\title{
Regionale Aspekte des Feststoffmanagements als Grundlage für den naturnahen Wasserbau im Mühlviertel und im Bayerischen Wald
}

\author{
Christoph Hauer · Sarah Höfler · Peter Flödl · Clemens Gumpinger · Helmut Habersack · Jacob Holzinger · \\ Alexander Kloibmüller · Patrick Leitner · Petr Lichtneger · Tobias Mayer · Franz Ottner · Daniela Riechl · \\ Beatrice Wagner · Torben Walter · Felix Weingraber · Wolfram Graf
}

Online publiziert: 20. Oktober 2016

(c) Der/die Autor(en) 2016. Dieser Artikel ist eine Open-Access-Publikation.

\begin{abstract}
Zusammenfassung Der naturnahe Wasserbau besitzt aufgrund seiner Begrifflichkeit bereits den Anspruch, natürliche und naturnahe Rahmenbedingungen und Prozesse in Planungen von wasserbaulichen Maßnahmen wie Hochwasserschutz oder Ufersicherungen miteinzubeziehen. Hier spielt in einer mittel- bis längerfristigen Betrachtung der Feststoffhaushalt eines Fließgewässers eine entscheidende Rolle. In Bezug auf natürliche oder anthropogene Veränderungen können Defizit und Überschuss $\mathrm{zu}$ entscheidenden und nachhaltigen Veränderungen der Lebensraumqualität führen. Vor allem in der Region der Böhmischen Masse ist ein Überschuss von Grobsand- und Feinkiesfraktionen (Korndurchmesser 1 bis $10 \mathrm{~mm}$ ) verantwortlich für regionale Probleme sowohl in Bezug auf den Hochwasserschutz als auch für die $\mathrm{Ge}$ wässerökologie, wie z. B. hinsichtlich des Erhalts von Kieslaichplätzen und Flussperlmuschelpopulationen. Im Forschungsprojekt „Feststoffmanagement

Wald“ konnte in umfangreichen Untersuchungen neben dem Ist-Zustand in acht ausgewählten Einzugsgebieten auch ein vertieftes Prozessverständnis hinsichtlich Ursachen und Wirkungszusammenhängen der kontinuierlichen Versandung gewonnen werden. Neben land- und forstwirtschaftlichen Aspekten wie der offenen Drainagierung von Wiesengräben oder dem Einfluss von Fichtenmonokulturen, konnten vor allem Aspekte der globalen Erwärmung (Zunahme der Starkniederschläge) oder des Sedimenttransportverhaltens für die zunehmende Degradierung des aquatischen Lebensraums bzw. die immer wiederkehrenden schutzwassertechnischen Anforderungen von Gewässerräumungen als Hauptursachen ausgemacht werden. Die aus dem hier vorgestellten Projekt gewonnenen Erkenntnisse bilden die Grundlage für zukünftige Maßnahmen des naturnahen Wasserbaus zur nachhaltigen Sanierung der Fließgewässer der Böhmischen Masse.
\end{abstract} im Mühlviertel und im Bayerischen

Priv.-Doz. DI Dr. C. Hauer $(\varangle)$.

DI P. Flödl, BSc. .

Univ.-Prof. DI Dr. H. Habersack

DI J. Holzinger, BSc.

DI A. Kloibmüller, BSc. .

DI Dr. P. Lichtneger - DI T. Mayer, BSc.

DI D. Riechl, BSc. · DI B. Wagner

Department für Wasser -

Atmosphäre - Umwelt, Institut für

Wasserwirtschaft, Hydrologie

und konstruktiven Wasserbau,

Universität für Bodenkultur Wien,

Muthgasse 107, 1190 Wien, Österreich

christoph.hauer@boku.ac.at

DI S. Höfler · DI C. Gumpinger

Blattfisch e.U. - Technisches

Büro für Gewässerökologie,

Gabelsbergerstraße 7, 4600 Wels,

Österreich
DI Dr. P. Leitner · Ass.-Prof. Dr. W. Graf Department Wasser - Atmosphäre Umwelt, Institut für Hydrobiologie und Gewässermanagement, Universität für Bodenkultur Wien, Max-Emanuel-Straße 17, 1180 Wien, Österreich

ao.Univ.-Prof. Mag. Dr. F. Ottner Department für Bautechnik und Naturgefahren, Institut für Geologie, Universität für Bodenkultur Wien, Peter-Jordan-Straße 70, 1190 Wien, Österreich

T. Walter, MA · Mag. F. Weingraber Abt. Oberflächengewässerwirtschaft, Amt der Oberösterreichischen Landesregierung,

Kärntnerstraße 10-12, 4021 Linz, Österreich
Schlüsselwörter

Feststoffmanagement .

Schutzwasserwirtschaft

Gewässerökologie · Klimawandel

Regional aspects of sediment management for near-natural river engineering in the Bohemian Massif

Abstract The aim of near-natural river engineering is to consider natural and near-natural framework conditions and processes in the planning of river engineering projects like flood protection or bank stabilization measures - a context in which the mid to long-term monitoring of sediment and sediment dynamics plays a central part.

With regard to natural and anthropogenic changes, deficits and surpluses of sediments can result in massive and sustainable changes in habitat quality of rivers. Especially in the region of the Bohemian Massif, a surplus of coarse sand and fine gravel (grain size 1 to 10 $\mathrm{mm}$ ) is the cause of regional problems, both in terms of flood protection and freshwater ecology.

Basic and applied research conducted in the project "Sediment management in the Mühlviertel and the Bavarian Forest" documented not only the status quo of eight selected catchment areas, but also yielded valuable insights into the causes and interrelations responsible for river bed degradation due to coarse sand inputs. In addition to agricultural and forest management aspects like the open drainage of meadow ditches or the influence of spruce monocultures, the impacts of global warming (increased frequency of heavy rains) and sediment transport dynamics were identified as the major causes of the increasing degradation of aquatic habitats and the need for an 
integrative sediment management in the investigated rivers.

The results presented in this paper deliver important information for future near-natural river engineering measures in order to restore rivers in the region of the Bohemian Massif.

Keywords Sediment management . Water conservation · Freshwater ecology $\cdot$ Climate change

\section{Einleitung}

Sowohl national als auch international wird der Zustand der Fließgewässerhydromorphologie als einer der entscheidenden Einflussfaktoren auf die Lebensraumqualität von aquatischen Organismen festgehalten (Ward und Stanford 1995; Dudgeon et al. 2006). Vor allem in Hinblick auf die Zielvorgaben der Europäischen Wasserrahmenrichtlinie (Eu, 2000: Directive 2000/60/EC) spielt der einzugsgebietsspezifische Feststoffhaushalt, als Rückgrat einer eigendynamischen Gewässerentwicklung, vor allem unter dem Aspekt der Nachhaltigkeit von Maßnahmen und Sanierungszielen eine entscheidende Rolle. Negative Auswirkungen auf die aquatische Fauna durch Veränderungen der Feststoffproduktion und des Feststofftransports in alpinen Einzugsgebieten sind häufig geprägt von einem Ungleichgewicht zwischen Transportkapazität und Sedimentverfügbarkeit auf Streckenebene mit der Konsequenz von Fließgewässereintiefung bis hin zum Sohldurchschlag bzw. Abpflasterung der Gewässersohle. In Abhängigkeit von der Geomorphologie können aber auch gegenteilige mittel- bis längerfristige Entwicklungen in Fließgewässern auftreten (z. B. Sedimentüberschuss), die den naturnahen Wasserbau wiederum vor spezielle Aufgaben eines nachhaltigen Feststoffmanagements stellt.

Beispielsweise zeigen das nördlich der Donau gelegene Mühlviertel bzw. auch weite Teile des Bayerischen Waldes aufgrund der geomorphologischen Rahmenbedingungen (Kristallin der Böhmischen Masse) einige flussmorphologische Besonderheiten bzw. spezielle Probleme im Vergleich zu alpinen Fließgewässern. Weite Strecken der Flüsse entsprechen dem sogenannten „Flachbettflusstyp“ ohne nennenswerte Strukturierung im Längsverlauf (z. B. Furt-Kolk-Sequenzen) bzw. Kiesbankstrukturen entlang der Ufer (Hauer et al. 2011; Hauer 2015). Die Deckschicht der Flusssohle in diesen Abschnitten ist dominiert durch die Korngrößen Meso- $\left(\mathrm{d}_{\mathrm{m}}=6\right.$ bis $\left.20 \mathrm{~cm}\right)$ aber auch Makrolithal $\left(\mathrm{d}_{\mathrm{m}}=20\right.$ bis $40 \mathrm{~cm}$ ). In den steileren kaskadenartigen Durchbruchsstrecken ist vor allem Megalithal $\left(\mathrm{d}_{\mathrm{m}}>40 \mathrm{~cm}\right)$ mit nur geringem Rundungsgrad der Sedimente typisch. Die teilweise geringe bordvolle Abflusskapazität ermöglicht selbst bei außergewöhnlichen Hochwasserereignissen (z. B. $\mathrm{HQ}_{100}$ ) in vielen Bereichen kaum ein Aufreißen der Deckschicht durch zu geringe Sohlschubspannungen aufgrund von Austauschprozessen (Energieverlusten) mit dem überfluteten Vorland. Weiters ist geeigneter Laichkies $\left(\mathrm{d}_{\mathrm{m}}=2\right.$ bis $\left.5 \mathrm{~cm}\right)$ limitiert und großteils in der Unterschicht gebunden und somit nicht als Laichsubstrat für Salmoniden (Salmo trutta, Thymallus thymallus) verfügbar (Abb. 1).

Spezifische Probleme in Zusammenhang mit dem Feststoffhaushalt entstehen in der Region Mühlviertel und Bayerischer Wald vor allem durch den zunehmenden Eintrag von "Granitgrus“ (Korngrößen 1 bis $10 \mathrm{~mm}$ ) aus den $\mathrm{Zu}$ - bringerbächen in die größeren Fließgewässersysteme. Obwohl diese Korngrößenklassen technisch den Grobsandund Feinkiesfraktionen zuzuordnen sind, wird hier von der Problemstellung der sogenannten „Versandung“ der Fließgewässer gesprochen. Vor allem in den Übergangsbereichen vom Hochland hin zu den Überflutungsflächen der Donau kommt es durch die Abnahme des Gefälles zu starken Verlandungstendenzen. Durch die damit einhergehende Verringerung der Abfuhrkapazität des Fließgewässers (oftmals in Kombination mit aufkommender Vegetation) besteht in diesen Bereichen ein zusätzliches Gefährdungsund Schadenspotenzial im Falle von Hochwasserereignissen, bis hin zu einer unmittelbaren Gefährdung des Hochwasserschutzziels $\left(\mathrm{HQ}_{100}\right)$. Die deshalb notwendigen Materialräumungen zur Gewährleistung eines dem Schutzziel entsprechenden Abflussquerschnittes in sehr kurzen Zeitabständen sind sowohl in wirtschaftlicher wie auch gewässerökologischer Hinsicht als nicht nachhaltig anzusehen. Um diese Entnahmen einschränken zu können, besteht die Notwendigkeit, den Materialeintrag flussauf nachhaltig zur reduzieren bzw. Möglichkeiten zu diskutieren, ob das Geschiebe mitunter gezielt weiter flussauf entnommen werden kann.

Neben den Anlandungen in schutzwassertechnisch relevanten Strecken kommt es auch zur Veränderung von hydromorphologischen Habitatstrukturen durch den verstärkten Grobsandund Feinkieseintrag $\left(\mathrm{d}_{\mathrm{m}}=1\right.$ bis $\left.10 \mathrm{~mm}\right)$ in naturnahen Fließgewässerstrecken (Abb. 2). Im Wesentlichen sind in den Fließgewässern des Mühlviertel und des Bayerischen Waldes drei flussmorphologische Typen dominierend. Großteils sind die Flüsse dem sogenannten
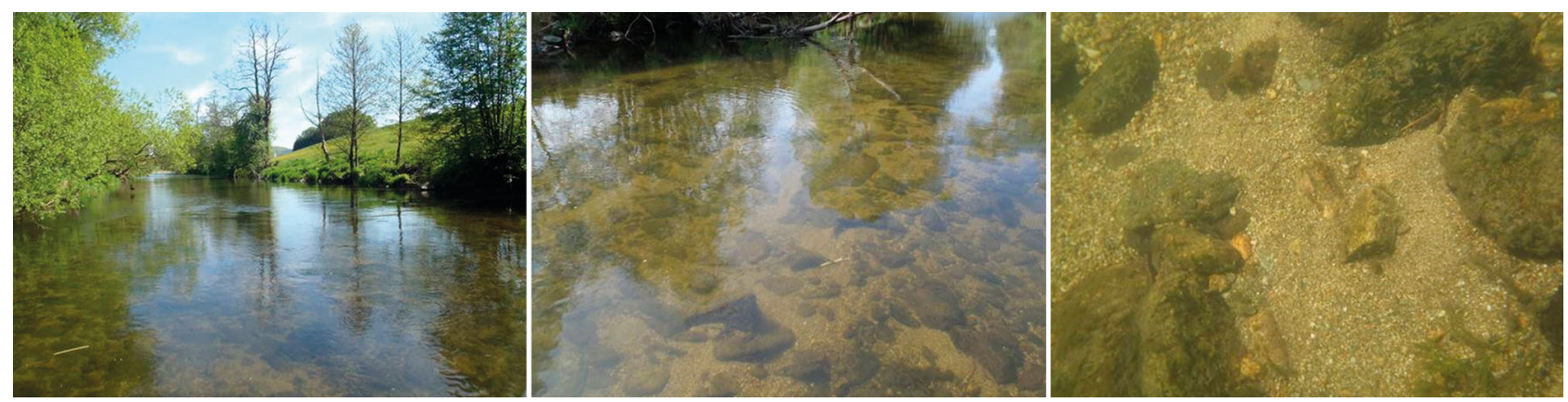

Abb. 1 Versandeter Bereich an der Großen Mühl im Gewässerabschnitt zwischen Aigen i. M. und Ulrichsberg; deutlich ist der Verschluss des Lückenraums durch die Sandfraktionen zu erkennen bzw. der geringe Rundungsgrad des Deckschichtmaterials in der Mesolithal-Kornfraktion 


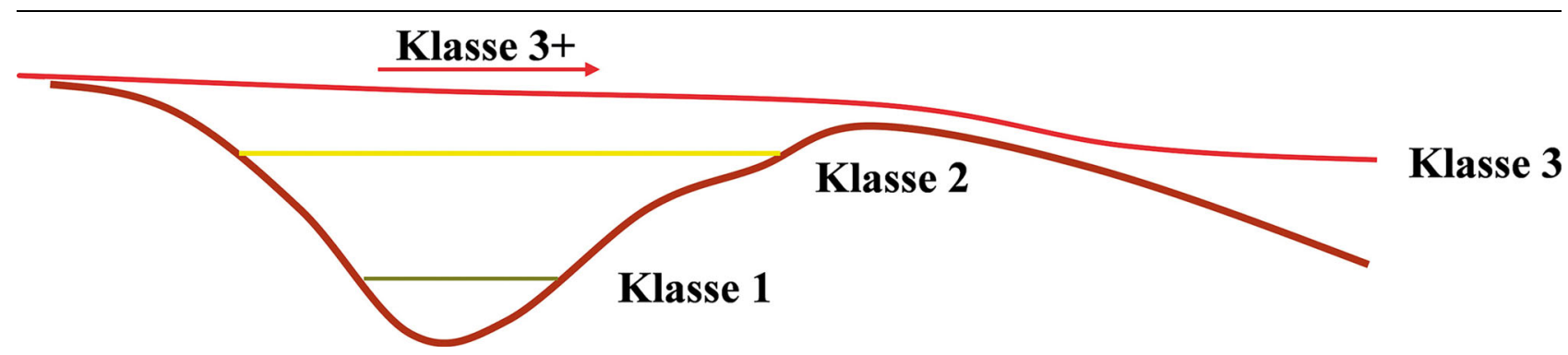

Abb. 2 Ausweisung der unterschiedlichen Versandungsklassen und ihre Auswirkung in Bezug auf die lokale Flussmorphologie (Furt-Kolk-Typ) (Hauer 2015)

Flachbetttyp zuzuordnen (Hauer et al. 2013). In den Verebnungsflächen mit mäandrierendem Flussverlauf finden sich aber auch Fließgewässerstrecken mit einer Furt-Kolk-Abfolge und in den Steilstufen zwischen den Verebnungsflächen Abschnitte des sogenannten Kaskadentyps (Hauer 2015). Vor allem in den Furt-Kolk- bzw. aber auch in den Flachbettstrecken kommt es durch die „Versandung“ mitunter zu einer deutlichen Veränderung der $\mathrm{Ge}$ wässermorphologie. In Bezug auf diese Veränderungen wurde eine eigene Klassifikation der „Versandungsgrade“ für das Projekt „Feststoffmanagement im Mühlviert und im Bayerischen Wald" entwickelt. In Abb. 2 ist beispielhaft die Klassifizierung der Versandungsgrade anhand eine Furt-Kolk-Abschnittes beschrieben. Die gravierendste Form der Veränderung ist eine vollständige Bedeckung der Gewässersohle mit den Verwitterungsprodukten von Granit und Gneis, inklusive eine Auffüllung vorhandener Kolkbereiche. Dieser Zustand wird mit Versandung Klasse 3 und Klasse $3+$ bezeichnet. Durch diese sedimentologische Überprägung kommt es mitunter $\mathrm{zu}$ wesentlichen Veränderungen in Bezug auf die Klassifizierung des flussmorphologischen Grundtyps. Fließgewässer, die ursprünglich als Flachbett- oder Furt-Kolk-Gewässerstrecken ausgewiesen wurden, zeigen nun geometrische und vor allem sedimentologische Eigenschaften des Dünentyps (Einteilung nach Montgomery und Buffington 1997). Eine falsche hydromorphologische Grundeinstufung von Fließgewässern ist dadurch möglich.

Weiters kommt es aus ökologischer Sicht neben dem Verlust bestimmter Habitattypen (z. B. Kolke, Abb. 2) auch zu einem Verschließen des Schotterlückenraums (Kolmation) als eine Form der kontinuierlichen und nachhaltigen Degradation der Gewässersohle als Lebensraum. Dabei sind auch die unterschiedlichen Auswirkungen von Feinsedimenten wie Schluff und Feinsanden im Vergleich zur beschriebenen Versandung auf die aquatische Zönose zu unterscheiden (siehe dazu Höfler et al. 2016, in diesem Heft). Während die sehr feinen Anteile durch Kolmationseffekte im hyporheischen Interstitial zu einem Sauerstoffmangel führen können (z. B. Eriksen 1966; Rosenberg und Wiens 1978; Cline et al. 1982; Culp und Davies 1985; Erman und Ligon 1988; Wood und Armitage 1997; Ofenböck et al. 2008), verursachen die Sandschichten durch ihre hohe Mobilität Probleme - vor allem für benthische Invertebraten. Beispielsweise konnte an der Aist in vergleichenden Untersuchungen festgehalten werden, dass in versandeten Bereichen (vollständig von Granitgrus bedeckt) eine Reduktion der BenthosBiomasse von > $80 \%$ im Vergleich $\mathrm{zu}$ morphologischen Referenzstellen nachzuweisen war (Leitner et al. 2015).

Aufgrund dieser schutzwasserwirtschaftlichen, morphologischen und gewässerökologischen Probleme wurde von Seiten des Landes Oberösterreich in Zusammenarbeit mit dem Bundesministerium für Land- und Forstwirtschaft, Umwelt und Wasserwirtschaft, dem Wasserwirtschaftsamt Deggendorf und dem Bayerischen Landesamt für Umwelt mit finanzieller Unterstützung durch das Interreg-Programm „Bayern - Österreich“ die Studie „Feststoffmanagement im Mühlviertel und im Bayerischen Wald" zur wissenschaftlichen Bearbeitung ausgeschrieben.

Ziel des Forschungsprojektes war es, in insgesamt elf Arbeitspaketen eine überregionale Betrachtung hinsichtlich Geschiebebewirtschaftung durchzuführen (Hauer et al. 2015). Neun der elf Arbeitspakete haben einen Schwerpunkt auf Grundlagenerhebungen und Prozessanalysen im Bereich schutzwasserwirtschaftlicher und ökologischer
Fragestellungen. Diese umfangreichen Untersuchungen bilden die Grundlage für den vorliegenden Fachbeitrag und sollen die Implementierung von zielgerichteten, interdisziplinären $\mathrm{Ma}$ nahmen für ein nachhaltiges Management des Versandungsproblems der Fließgewässer im Mühlviertel und im Bayerischen Wald ermöglichen.

\section{Untersuchungsgebiet}

Insgesamt wurden acht Fließgewässer der Böhmischen Masse in Hinblick auf den schutzwasserwirtschaftlichen, sedimentologischen und ökologischen Ist-Zustand bzw. die maßgeblichen Prozesse und Ursachen für die Versandung untersucht. In Österreich waren dies von Ost nach West aufgelistet: Naarn, Aist (Wald- und Feldaist), Gusen (Große und Kleine Gusen), Rodl sowie die Große und Kleine Mühl (Abb. 3). In Bayern wurden im Zuge der Feststoffmanagement-Studie die Fließgewässer Osterbach und Erlau untersucht (Abb. 3).

\section{Methodik}

\subsection{Grundlagenerhebungen}

Ein wesentlicher Teil der Arbeiten im Forschungsprojekt „Feststoffmanagement im Mühlviertel und Bayerischer Wald" war die Ist-Bestandsanalyse des hydromorphologischen Grundzustands bzw. die Erfassung der Belastungen durch den Feststoffeintrag in den einzelnen Flussgebieten. Die dafür notwendigen Untersuchungen im Freiland erfolgten im Wesentlichen in zwei Teilarbeitsschritten. Zum einen wurde eine umfangreiche Beprobung der Gewässersohle in allen acht untersuchten Fließgewässern durchgeführt (Abb. 3). Zum anderen wurden alle Fließgewässer mittels eines eigens für das Projekt 


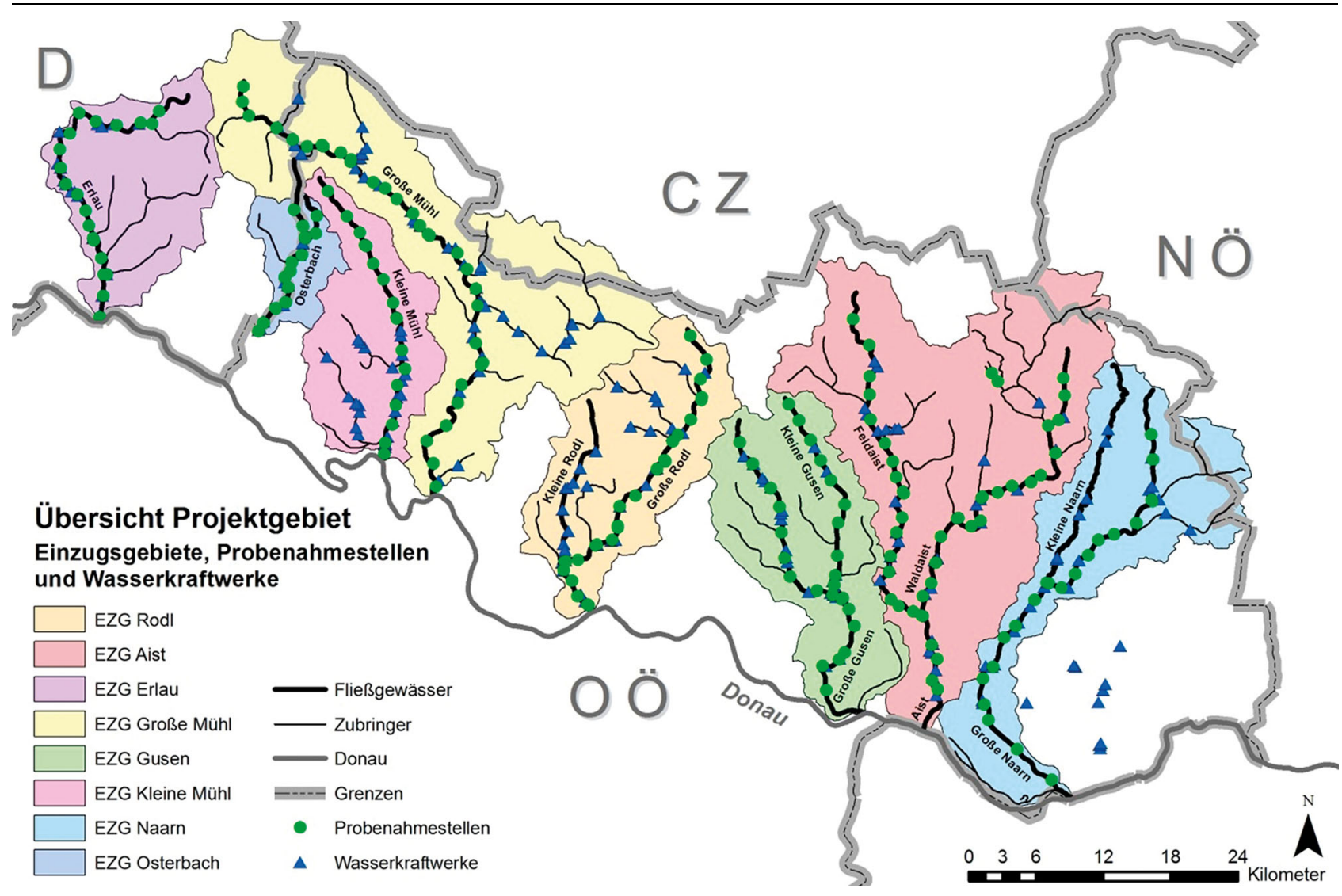

Abb. 3 Übersicht über die im Projekt untersuchten Fließgewässer $(n=8)$; inkludiert sind die flächige Ausweisung der unterschiedlichen Einzugsgebiete sowie die Flussstrecken und Sedimentprobenahmestellen (grüne Kreise)

entworfenen Bewertungsbogens kartiert.

\subsubsection{Korngrößenanalysen}

Die volumetrische Entnahme von Deckschicht- und Unterschicht-Proben (Abb. 3) erfolgte in sogenannten Flachbett-Abschnitten mit einem durchschnittlichen Gefälle von 3 bis $5 \%$ bzw. in den Kolk-Furt-Bereichen mit einem Gefälle $<1,5 \%$. In den Steilstufen zwischen den Verebnungsflächen bzw. in Bereichen flussauf der großen Donaubecken, mit einem Gefälle $>1 \%$, wurde die Korngrößenverteilung semiquantitativ mittels Kluppenmaße und Abschätzung der Feinanteile durchgeführt. Die Kluppenproben fanden jedoch keinen Eingang in die statistischen Analysen der Korngrößen.

Die volumetrische Probennahme erfolgte in Form eines standardisierten Verfahrens (USGS) in dem ein $60 \times 60 \mathrm{~cm}$ großer Rahmen (Höhe $20 \mathrm{~cm})$ am jeweiligen Probenahmepunkt auf die Gewässersohle aufgesetzt wurde. Innerhalb des strömungsberu- higten Bereichs wurden die Sedimente getrennt nach Deck- und Unterschicht mechanisch entnommen, wobei auch Feinanteile $<1 \mathrm{~mm}$ quantitativ erfasst wurden. Um einer möglichen Variabilität der Korngrößenzusammensetzung im Gewässerquerschnitt Rechnung zu tragen, wurden an den Untersuchungsstellen Proben sowohl rechts als auch links der Flussachse genommen. Somit konnten vor allem auch in das Fließgewässer einmündende Zuflüsse und ihre Auswirkungen auf den Sedimenthaushalt berücksichtigt werden.

Die Sedimentproben wurden nach Trocken und Sieben in Form von Kornverteilungskurven für weitere statistische Analysen aufbereitet. Ausgewertet wurden charakteristische Korngrößen wie $d_{50}$ (50\% feiner), $d_{m}$ (geometrisches Mittel) und $d_{90}(90 \%$ feiner). Weiters wurden hinsichtlich der gezielten Auswertung bzw. Bewertung der Feinsedimente (Korngrößen $<1 \mathrm{~mm}$ ), ein wesentlicher Teil der Sedimentproben ( $\mathrm{n}=459$ ), an der Kornfraktion von $2 \mathrm{~cm}$ geteilt und diese Feinanteile eigens hinsichtlich der Ton- bzw. Schlufffrak- tionen bewertet $(<0,125 \mathrm{~mm})$. Diese Aufteilung der Korngrößenfraktionen war notwendig, um eine mögliche prozentuelle Verschiebung der Feinanteile durch anteilsmäßig hohe Grobkornfraktionen auszuschließen. Durch die Aufteilung konnte somit ein objektiv vergleichbarer Datensatz generiert werden.

Die Ergebnisse der Bestimmung der verschiedenen Korngrößenparameter aus Einzelproben wurden mit abschnittsbezogenen bzw. einzugsgebietsbezogenen Größen verschnitten. Als abschnittsbezogene Größe wurde das lokale Sohlgefälle (-) im Abschnitt der Probennahme herangezogen. Das Sohlgefälle wurde durch die Auswertungen des Längenschnitts (Grundlage Laserscan/DORIS, Auflösung $1 \times 1 \mathrm{~m}$ ) bestimmt. Für die Berechnung des lokalen Gefälles wurde ein Abschnitt $100 \mathrm{~m}$ flussauf und $100 \mathrm{~m}$ flussab des Probenahmepunktes bewertet. Da Proben nur in freien Fließstrecken und nicht in Rückstaubereichen von Querbauwerken entnommen wurden, ist von einem minimalen Fehler der Laserscandaten 


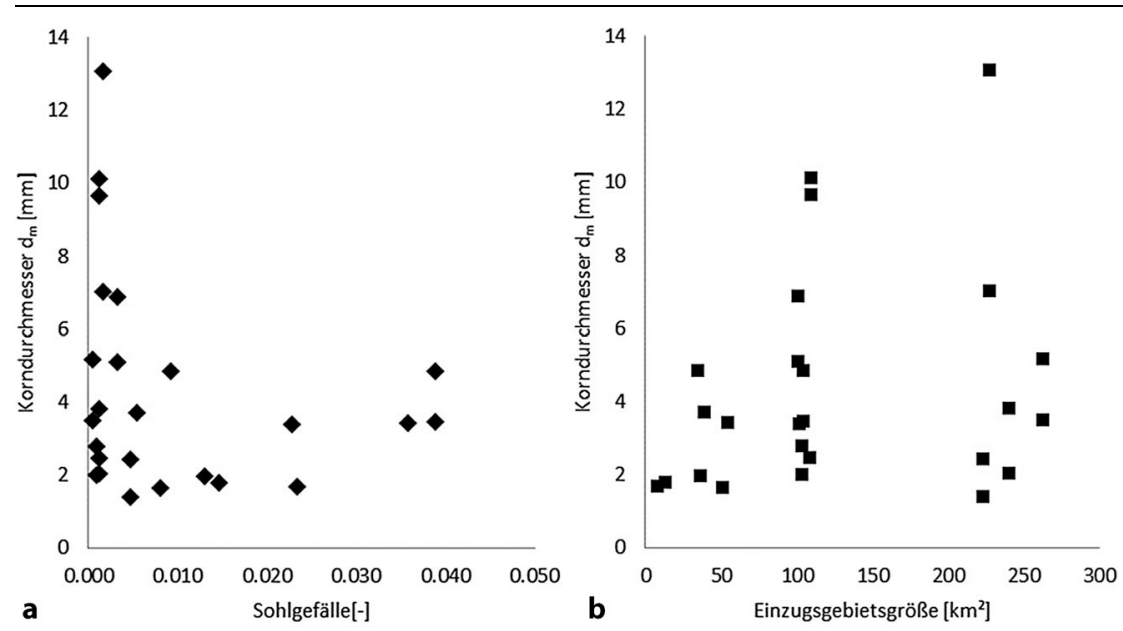

Abb. 4 Auswertung Siebanalyse (Gusen, Mischprobe): Gegenüberstellung maßgebender Korndurchmesser $\mathrm{d}_{\mathrm{m}}$ in mm und Sohlgefälle (dimensionslos); (b) Auswertung Siebanalyse (Gusen, Mischprobe): Gegenüberstellung maßgebender Korndurchmesser $\mathrm{d}_{\mathrm{m}}$ in mm und Einzugsgebietsgröße in $\mathrm{km}^{2}$

in Bezug auf das Sohlgefälle auszugehen. In Summe wurden für sieben der acht untersuchten Flüsse detaillierte Längenschnitte erstellt. Für die Erlau (Bayern) konnten keine vergleichbaren Datengrundlagen im Projekt verwendet werden. Als einzugsgebietsbezogene Größe wurde die Fläche der Entwässerung flussauf des Probenahmepunktes angegeben. Diese Teileinzugsgebietsflächen wurden mittels GIS-Analysen (Arc-GIS Watershed) basierend auf dem 1 x 1 m-Höhenmodell des Untersuchungsgebietes berechnet.

\subsubsection{Kartierung des Ist-Zustands/ naturräumliche Erhebung}

Die Definition des Erhebungsstandards wurde im Zeitraum Dezember 2013 bis Juli 2014 umgesetzt. Aus den umfangreichen Erfahrungen aus anderen Projekten und vorhandenen nationalen und internationalen Erhebungsstandards (vgl. z. B. BMLFUW 2010; LAWA 2000) wurden die möglichen Parameter für eine Vor-Ort-Erhebung zusammengetragen. Deren Anwendbarkeit sowie Aussagekraft wurde bei gemeinsamen Probekartierungen überprüft.

Der Kartierbogen umfasst neben hydromorphologischen Grundparametern, Angaben bezüglich der Choriotopverteilung, hochwasserschutzrelevante Angaben wie beispielsweise die Möglichkeit von Materialmobilisierungen in den Uferbereichen, gewässerökologische Einstufungen und als wichtigsten Punkt die Versandungs-Klassifizierung. Diese wurde für die Kartierung im Freiland für die unterschiedlichen Flusstypen abgegrenzt. Darüber hinaus ergibt sich eine flächenmäßige Abschätzung des Versandungsgrades durch die Aufnahme der Choriotoptypen. Hinsichtlich der Auswirkungen auf die aquatische Fauna und Flora, und im Speziellen auf die Flussperlmuschel, wurde bei den Freilandarbeiten auf die Unterscheidung zwischen Versandung und Feinsediment-Problematik Wert gelegt. Der Grad an Feinsedimentbelastung wurde angelehnt an die Erkenntnisse aus einer Studie zu diesem Thema (vgl. Höfler und Gumpinger 2014) abgegrenzt.

Die Aufnahmen im Freiland erfolgten im Winter 2014/15. Die Gewässer wurden jeweils von der Mündung flussauf kartiert. Die Abschnittsgrenzen sowie punktuelle Informationen wurden mittels GPS-Geräten aufgenommen. Des Weiteren sammelten die Kartierteams umfangreiches Fotomaterial, um Situationen und Prozesse zu dokumentieren. Insgesamt umfasst die Kartierung über 438,2 Flusskilometer. Die erhobenen Daten wurden in eine dafür angelegte Access-Datenbank aufgenommen, aus der nach entsprechender Sichtung und Prüfung der Daten Shapefiles in ArcGIS exportiert wurden.

\subsection{Schutzwasserbauliche Aspekte}

In der Einleitung des vorliegenden Fachbeitrags wurden bereits die schutzwasserwirtschaftliche Problematik und die Herausforderungen für den Naturnahen Wasserbau durch die Versan- dung der Fließgewässer der Böhmischen Masse angesprochen. Neben der bereits angeführten Veränderung der Gewässergeometrie (Reduktion des Abflussquerschnitts) an Gefällsknicken, beispielsweise an den Übergängen des Mühlviertler Hochlandes in die Donauebene, besitzt auch die dynamische Komponente des Feststofftransports einen schutzwasserwirtschaftlichen Aspekt. Durch die Umwandlung der Bewegungsenergie des Wassers in Transportenergie des Sohlmaterials kommt es zu einer Reduktion der Fließgeschwindigkeit. Dies kann durch die Kontinuitätsgleichung erklärt werden und führt mitunter zu Aufspiegelungen verglichen mit einem Abschnitt ohne dynamische Sohlkomponente. Die Auswirkungen des Geschiebetriebs bei Hochwasser wurden im vorliegenden Projekt quantitativ nicht im Detail untersucht, es gibt jedoch diesbezügliche Studien von Fließgewässern aus dem alpinen Raum (Neuhold et al. 2009). Im gegenständlichen Fall wurde vor allem auf die querschnittsverändernden Komponenten der Feststoffdynamik mittels hydrodynamisch-numerischer Modellierung eingegangen.

\subsubsection{Detaillierte schutzwasserwirtschaftliche Analysen im Aist-Einzugsgebiet}

Im Aist-Einzugsgebiet wurden vertiefende Untersuchungen hinsichtlich des Versandungsrisikos als Grundlage für ein zukünftiges Management durchgeführt. Die Ausweisung der Risikobereiche für eine mögliche Versandung wurde für alle Fließgewässer mit einer Einzugsgebietsgröße $>10 \mathrm{~km}^{2}$ im Einzugsgebiet der Aist mittels eines eindimensionalen hydrodynamischnumerischen (HN) Feststofftransportmodells ermittelt (basierend auf DHM Grundlage Laserscan/DORIS, Auflösung 1 x $1 \mathrm{~m})$. Weiters wurde als Grundlage für die detaillierte schutzwasserwirtschaftliche Analyse eine GISbasierte Analyse der Landnutzung, der Geologie und des Erosionsrisikos in den einzelnen Teileinzugsgebieten des AistSystems durchgeführt.

\subsection{Gewässerökologische Untersuchungen}

Aus gewässerökologischer Sicht wurden im vorliegenden Forschungsprojekt drei Themen inhaltlich behandelt. Untersucht wurden im Detail die Aus- 


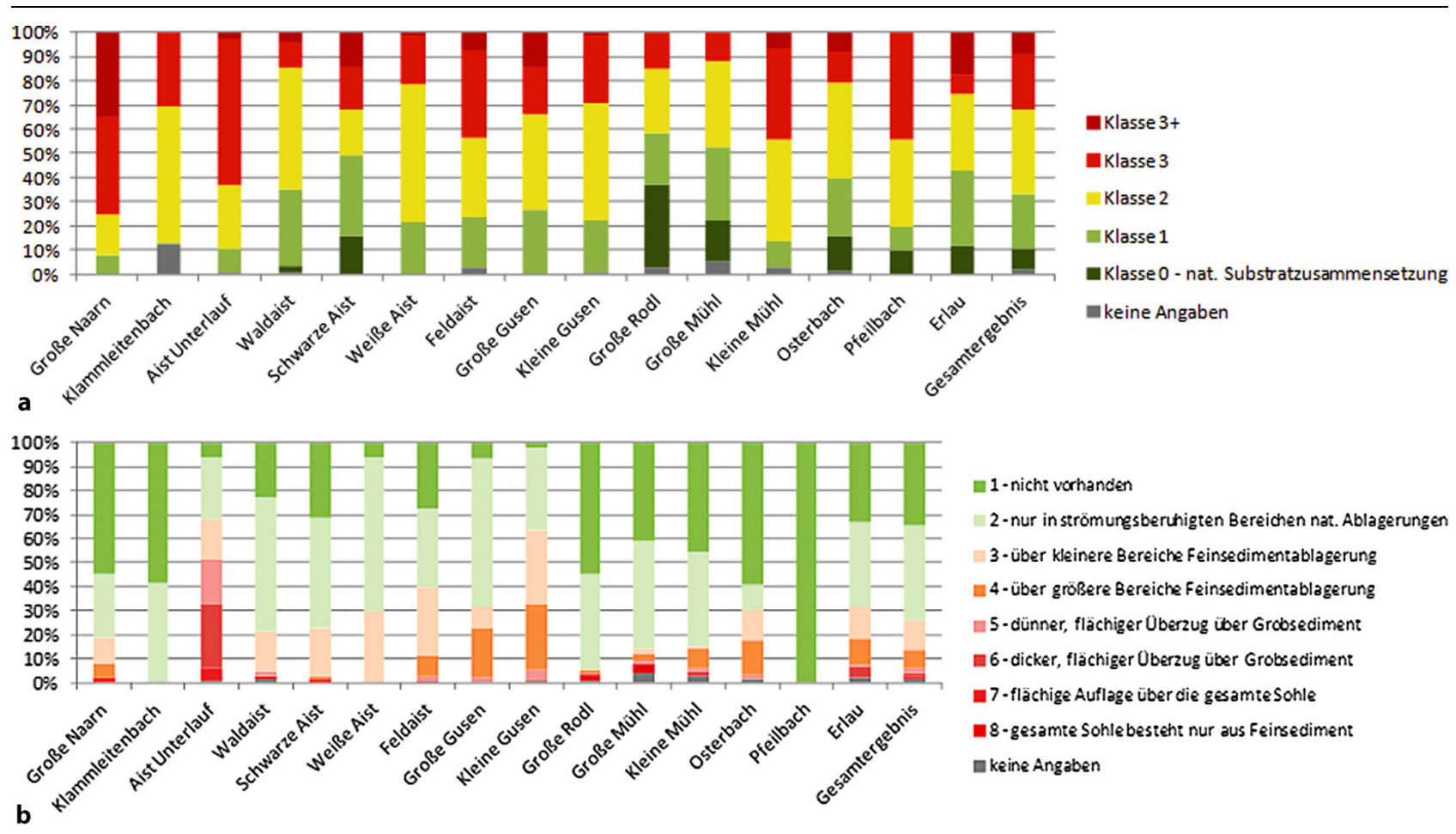

Abb. 5 (a) Darstellung der Versandungsklassen in den untersuchten Fließgewässern, (b) Darstellung der Feinsedimentausdehnung in den untersuchten Fließgewässern

wirkungen der Versandung bzw. unterschiedlicher Versandungsklassen in Bezug auf die Quantität und Qualität des Makrozoobenthos (MZB). Dazu wurden im Zeitraum von 14. bis 16 . April 2014 an den Gewässern Große Mühl, Große Rodl, Gusen, Kleine Mühl und Osterbach in Referenzabschnitten und in Abschnitten mit unterschiedlicher Versandung MZB-Proben entnommen. Lediglich an der Kleinen Mühl wurde kein Referenzabschnitt beprobt. Das Untersuchungsdesign umfasst somit 12 Untersuchungsstellen, davon 5 in Referenzbereichen und 7 in durch Versandung beeinflussten Abschnitten. An jeder Untersuchungsstelle wurden je eine Multi-Habitat-Sampling-Probe (MHS) und in unterschiedlicher Anzahl habitatspezifische Einzelproben entnommen.

Weiters wurden die erhobenen Daten der Freilandaufnahmen mit vorhandenen Daten bezüglich der Flussperlmuschel (Margaritifera margaritifera) verschnitten, um so Erkenntnisse über die Qualität und Belastung des Flussperlmuschellebensraums zu gewinnen. Bei diesen Analysen wurde neben einer Bewertung der negativen Auswirkung eines übermäßigen Eintrags von Granitgrus auch der mögliche Einfluss von feineren Sedimenten $(<0,125 \mathrm{~mm})$ un- tersucht. Für vertiefende Analysen wurden Muscheldaten (Funde und NichtFunde) in den Einzugsgebieten von Erlau, Kleiner und Großer Mühl sowie Waldaist herangezogen, da hier flächendeckende Daten über die Bestände im Untersuchungsgebiet vorliegen.

Aus fischökologischer Sicht wurden aus parallel laufenden und bereits abgeschlossenen Projekten bedeutende Aspekte der Laichplatzqualität gemeinsam mit den Ergebnissen des Projekts diskutiert, welche in der Entwicklung bestimmter Maßnahmentypen berücksichtigt wurden.

\section{Ergebnisse}

\subsection{Naturräumliche Erhebungen}

\subsubsection{Korngrößenanalysen}

Die Ergebnisse der durchgeführten statistischen Analysen in Bezug auf die volumetrischen Sedimentproben ( $n=459$ ) zeigten in den meisten Analysen keinen Zusammenhang zwischen Sohlgefälle (-) bzw. EZG-Größe $\left(\mathrm{km}^{2}\right)$ und der Variabilität charakteristischer Korngrößen (z. B. $d_{m}$ ) bzw. den Grad der Versandung (Abb. 4a und b). Grundlage für die Ermittlung eines möglichen $\mathrm{Zu}$ sammenhangs bildete ein lineares Re- gressionsmodell. Ausnahmen, die einen signifikanten Zusammenhang zeigten, waren die Auswertungen der Proben der Kleinen Mühl $\left(\mathrm{R}^{2}=0,80\right)$, wobei der Stichprobenumfang $(n=7)$ in diesem kleinen System aber gering war.

\subsubsection{Kartierung des Ist-Zustands/ Naturräumliche Erhebung}

Auf Einzugsgebietsebene betrachtet, können aufgrund der Kartierungsergebnisse (438,2 Flusskilometer) deutlich zwei Gruppen unterschieden werden: die wenig versandeten Systeme der Erlau, der Großen Mühl und der Rodl und die eher stark belasteten anderen Flusssysteme. Doch auch bei diesen Fließgewässern gibt es mitunter große lokale Unterschiede hinsichtlich der dokumentierten Versandungsgrade. Am auffälligsten versandet sind:

- das gesamte Naarn-System,

- Feldaist,

- Aist-Unterlauf,

- Kleine Gusen,

- Gusen-Unterlauf und

- Kleine Mühl.

In Abb. 5 sind die Ergebnisse der Kartierung hinsichtlich (a) Versandungsklassen bzw. auch (b) hinsichtlich der Feinsedimentbelastung (Ton, Schluff 


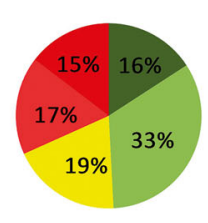

- Klasse 0 - nat. Substratzusammensetzung

Klasse 1

Klasse 2

Klasse 3

- Klasse 3+

keine Angaben

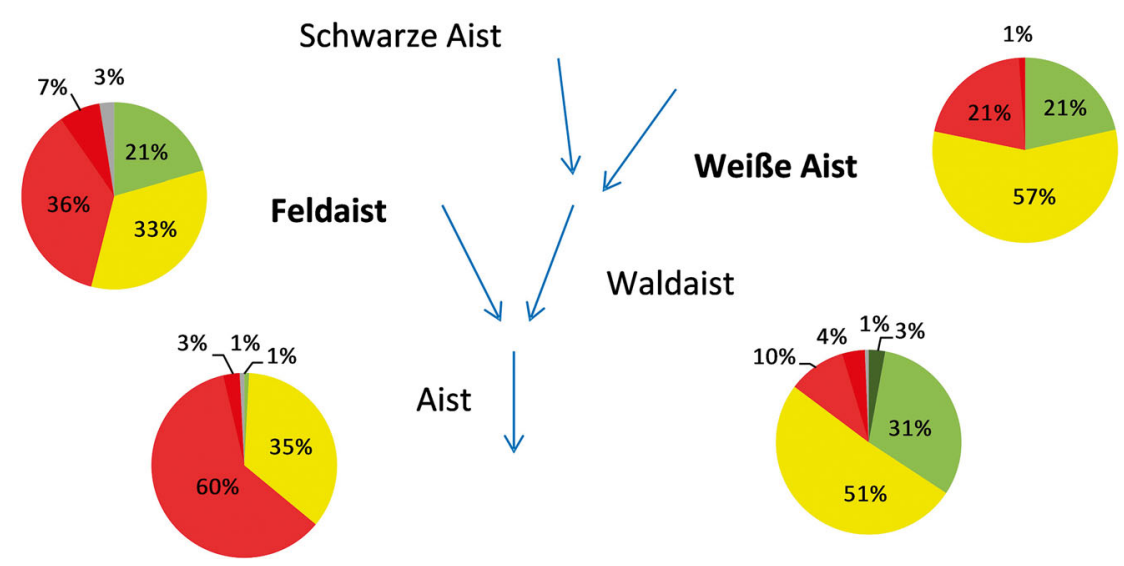

Abb. 6 VariableVersandungsgrade in Teileinzugsgebieten bzw. -abschnitten des AistSystems

bis Feinsandfraktionen) je nach untersuchtem (Teil-)Flussgebiet gegenübergestellt. Als ein Beispiel zur Illustration der oftmals lokal sehr unterschiedlichen Bedingungen kann das Aist-System herangezogen werden (Abb. 6). Je nach Geologie und anthropogener Überprägung im Einzugsgebiet ergeben sich sehr unterschiedliche Sedimentbedingungen in den Gewässern. Eindeutig nachgewiesen wurde auch der $\mathrm{zu}$ erwartende negative Zusammenhang zwischen der Einschätzung der Eignung einer Strecke für die kieslaichende Fischfauna und dem Versandungsgrad. Vor allem in der Äschenregion (Hyporhithral) treffen umfangreiche menschliche Überprägungen, die $\mathrm{zu}$ einer stark beeinträchtigten Sedimentsituation führen, auf eine vergleichsweise sensible aquatische Fauna. Auch der positive Einfluss von strukturgebendem Grobgeschiebe konnte eindeutig in den Daten nachgewiesen werden.

\subsection{Schutzwasserbauliche Aspekte}

Die angesprochenen Kornfraktionen des verwitternden Grundgesteins der Böhmischen Masse $\left(\mathrm{d}_{\mathrm{m}}=1\right.$ bis $\left.10 \mathrm{~mm}\right)$ fallen in jenen Bereich von Korngrößen, der laut Hjülström (1935) am mobilsten von allen Fraktionen ist. Der Bewegungsbeginn von Grobsand und Feinkies liegt bereits bei teilweise sehr geringen Fließgeschwindigkeiten. Die Mobilität der abgelagerten Sande z. B. in Bereichen lokaler Aufweitungen wird jedoch mitunter durch aufkommende Vegetation eingeschränkt. Beides zusammen, Ablagerungen bzw. böschungsstabilisierende Vegetation führen dazu, dass auch Fließgewässer der Böhmischen Masse eine sogenannte Regimebreite in Bezug auf den Abflussquerschnitt entwickeln. Als Regimebreite wird die Entwicklung eines bestimmen Gewässerquerschnitts in Abhängigkeit von unterschiedlichen physikalischen Einflussgrößen verstanden, ohne signifikante Störungen durch außergewöhnliche Hochwasserereignisse. Leopold et al. (1964) definierten in Summe acht Einflussgrößen, die letztendlich die Ausbildung einer bestimmten Gewässergeometrie bzw. eines bestimmten Fließgewässertyps bedingen. Neben den geometrischen Eigenschaften (1) bordvolle Breite, (2) bordvolle Tiefe und (3) Sohlgefälle werden auch sedimentologische Eigenschaften wie (4) der maßgebliche Korndurchmesser $\mathrm{d}_{\mathrm{m}}$ (= geometrisches Mittel der Kornverteilungskurve) in ihrer Bedeutung hervorgehoben bzw. wird (5) der quantitative Sedimenteintrag aus den flussauf gelegenen Einzugssystemen als beeinflussender Faktor genannt. Hinzu kommen hydrologisch-hydraulische Komponenten wie (6) die Abflussgröße (z. B. durchschnittlicher jährlicher Hochwasserabfluss) und die daraus resultierenden (7) Fließgeschwindigkeiten. Die einzelnen Größen sind teils wechselseitig beeinflusst. Somit resultiert auch die (8) Rauigkeit eines Fließgewässers aus der Kombination von Sedimentgröße und -sortierung (Kornrauigkeit) und der Gewässergeometrie (Formrauigkeit) in Abhängigkeit vom Durchfluss (Veränderung der relativen Rauigkeit).

In Bezug auf die Entwicklung von Regimebreiten gibt es eine Vielzahl veröffentlichter internationaler Studien (Leopold und Maddock 1953; Nixon et al. 1959; Kellerhals 1967), welche basierend auf Freilanderhebungen die Entwicklung bzw. Berechnungen von querschnittsbezogenen Größen wie bordvolle Breite $\left(\mathrm{Q}_{\mathrm{bf}}\right)$ oder bordvolle Tiefe $\left(\mathrm{T}_{\mathrm{bf}}\right)$ beinhalten. Bezugsgröße in diesen empirisch abgeleiteten Formeln ist meist der ein- bis zweijährliche Hochwasserabflusswert $\left(\mathrm{HQ}_{1}\right.$ oder $\left.\mathrm{HQ}_{2}\right)$ einer längeren hydrologischen Zeitreihe.

Einige der angeführten bekannten funktionalen Beziehungen wurden hinsichtlich ihrer Anwendbarkeit in den untersuchten Fließgewässern der Böhmischen Masse bewertet. Als Grundlage dienten tachymetrische Aufnahmen (Leica TC805) von Fließgewässerquerschnitten in lokalen, für den Hochwasserschutz aufgeweiteten Bereichen, welche bereits deutliche Verlandungen und teilweise stabilisierte Verhältnisse durch aufkommende Vegetation zeigten. Die Abschnitte lagen im Zuständigkeitsbereich der Gewässerbezirke Linz und Grieskirchen. Die Ergebnisse der Anwendung der unterschiedlichen Regimeformeln zeigten jedoch keine Übereinstimmung mit den sich entwickelnden Gewässerbreiten.

Aufgrund der umfangreichen Freilanderhebung konnten, neben den Vergleichen mit bestehenden Ansätzen, auch im Projekt selbst Analysen hinsichtlich der eigendynamischen quasistabilen Entwicklung von Gewässerbreiten durchgeführt werden. Es wurden diesbezüglich die aufgenommenen Gewässerbreiten dem lokalen Sohlgefälle und dem Mittelwasserabfluss (MQ) in den untersuchten Gewässerabschnitten $(n=6)$ gegenübergestellt. Die Ergebnisse zeigten sehr deutlich, dass die Mittelwasser-Abflussgröße mit der Entwicklung einer bestimmten Gewässerbreite korrespondiert (Flödl 2015).

Diese Abweichung, verglichen mit den bisher bekannten und verwendeten Regimeformeln, ist zu einem wesentlichen Teil dadurch begründet, dass die Grobsand- und Feinkiesfraktionen 


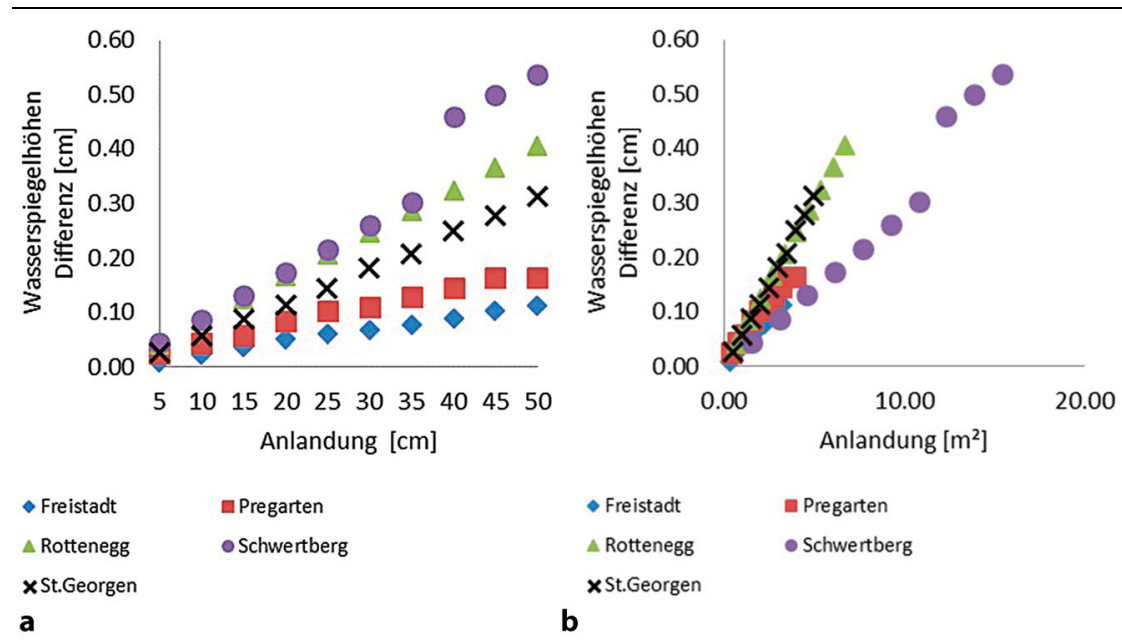

Abb. 7 (a) Vergleich der Anlandungsszenarien-Analyse bei jeweiligem HQ ${ }_{10}$-Abfluss. Dargestellt ist die Änderung der Wasserspiegellage in $\mathrm{cm}$ (Y-Achse) abhängig von der Anlandungshöhe in cm (X-Achse). Legende: Freistadt (blau), Pregarten (rot), Rottenegg (grün), Schwertberg (violett) und St. Georgen (schwarz); (b) Vergleich der Anlandungsszenarien-Analyse bei jeweiligem $\mathrm{HQ}_{10}$-Abfluss. Dargestellt ist die Änderung der Wasserspiegellage in $\mathrm{cm}\left(\mathrm{Y}\right.$-Achse) abhängig von der Anlandungsfläche in $\mathrm{m}^{2}$ (X-Achse). Legende: Freistadt (blau), Pregarten (rot), Rottenegg (grün), Schwertberg (violett) und St. Georgen (schwarz)

bereits bei deutlich geringeren Abflussgrößen als dem einjährlichen Hochwasser $\left(\mathrm{HQ}_{1}\right)$ mobil sind. Aus diesem Grund wird die Geometrie des Gewässerquerschnitts in Bereichen einer lokalen Überbreite bereits bei Abflüssen um Mittelwasser morphologisch verändert. Diese Erkenntnis hat mitunter eine wesentliche Bedeutung für Planung und Umsetzung zukünftige Hochwasserschutzmaßnahmen in der Region. Durch die gezielte Konzipierung und Ausführung einer Mittelwasser-Rinne in Bereichen kann der Weitertransport von einem Großteil der Grobsand- und Feinkiesfraktionen gewährleistet werden.

Weitere im Projekt „Feststoffmanagement im Mühlviertel und im Bayerischen Wald" untersuchte schutzwassertechnische Aspekte waren die Quantifizierung der Auswirkungen eines veränderten Gewässerquerschnitts in Bezug auf Veränderungen des Wasserspiegels bei mittleren und hohen Abflüssen. Die Analysen wurden in sechs schutzwassertechnisch relevanten Detailstrecken der Gewässerbezirke Linz und Grieskirchen durchgeführt. Für die Analysen wurden kalibrierte eindimensionale hydrodynamisch-numerische Modelle (HEC-RAS) verwendet. Einige ausgewählte Ergebnisse für einen 10jährlichen Hochwasserabfluss sind in den Abb. 7 ( $a$ und b) dargestellt.

\subsubsection{Detaillierte schutzwasserwirtschaftliche Analysen im Aist-Einzugsgebiet}

Die Ergebnisse der ermittelten Risikobereiche für eine potenzielle Versandung, basierend auf den hydraulischen Berechnungen in mehr als $11.000 \mathrm{Ge}-$ wässerquerschnitten mit einem mittleren Profilabstand von $25 \mathrm{~m}$, sind in Abb. 8 dargestellt. Laut Auswertung der Risikobereiche weisen die Aist und auch die Feldaist gegenüber der Waldaist ein viel höheres Potenzial hinsichtlich einer Versandung auf. Diese Aussage steht jedoch im Widerspruch zu den GISAnalysen in Bezug auf das Eintragspotenzial von Granitgrus. Dabei wurde die Waldaist mit einem deutlich höheren Potenzial bewertet als die Feldaist. Diese Unterschiede sind vor allem hydraulisch begründet, da durch ein geringeres Gefälle und die sehr deutliche menschliche Überprägung der Flussmorphologie der Feldaist die Transportkapazitäten im Fluss reduziert sind. Die Anteile an berechneten Risikobereichen an der Feldaist sind somit etwas höher als bei der Waldaist (Abb. 8). Besonders in flacheren Abschnitten treten verstärkt Risikobereiche auf. Dies ist auch am Jaunitzbach deutlich sichtbar, der durchgehend ein eher flaches einheitliches Gefälle $(0,67 \%)$ aufweist, weshalb auch über $70 \%$ der Fließgewässerlänge als Risikobereich ausgewiesen wurden.
Auf Basis der umfangreichen Kartierungen in allen untersuchten Fließgewässern im Projektsgebiet $(n=8)$ konnte an der Aist eine Verifizierung der Modellierungsergebnisse durchgeführt werden. Die Verifizierung erfolgte durch eine Überlagerung der Risikobereiche für eine Versandung mit jenen Abschnitten, die laut Kartierung Akkumulationen von Grobsand und Feinkies in unterschiedlichen Kategorien (Klasse 2 bis 3+) zeigten. In Bezug auf die sehr hohen Belastungen (Klasse 3 und $3+)$ zeigten die Modellierungsergebnisse für alle drei untersuchten Einzugsgebiete zusammengefasst eine Übereinstimmung von über $60 \%$. Wurden die Ergebnisse der Versandungsklasse 2 hinzugenommen, konnte eine Übereinstimmung zwischen Kartierung und Modellierung von über $80 \%$ ausgewiesen werden. Im detaillierten Vergleich zeigten die Ergebnisse im Bereich der Feldaist und Aist eine größere Übereinstimmung als in den untersuchten Fließgewässerabschnitten der Waldaist.

Die geringe Übereinstimmung im Bereich der Waldaist ist mit hoher Wahrscheinlichkeit durch die Verwendung des Laserscans in der Erstellung der Flussgeometrie begründet. Aufgrund des durch den Infrarot Laser verursachten Sohlfehlers (misst nur bis zur Wasseroberfläche) kann es mitunter zu einer Überschätzung der Risikobereiche kommen. Im Gegensatz dazu wurden für die Flüsse Aist bzw. Feldaist bis etwa Kefermarkt die Geländedaten aus dem erhaltenen SMS-Geländemodell der 2D-Abflussuntersuchungen entnommen, das auch auf terrestrisch vermessenen Gewässerquerschnitten und nicht nur auf Laserscandaten basiert.

\subsection{Gewässerökologie}

Am deutlichsten zeigt sich die Auswirkung von Versandungsstress in Bezug auf die durchgeführten MZB-Analysen anhand der Biomasse-Mittelwerte der habitatspezifischen Proben, hochgerechnet auf die geschätzte Substratverteilung in den unterschiedlichen Versandungsklassen. Demnach kommt es zu einem Zusammenbruch der errechneten Gesamt-Biomasse ab der Versandungsklasse 3 auf ca. 1/3 der Referenzsituation (Abb. 9).

Die Studie zeigt, dass erhöhter Feinsedimenteintrag nachhaltig negative Effekte auf aquatische Biozönosen hat. Die Verschlechterung der ökologischen 


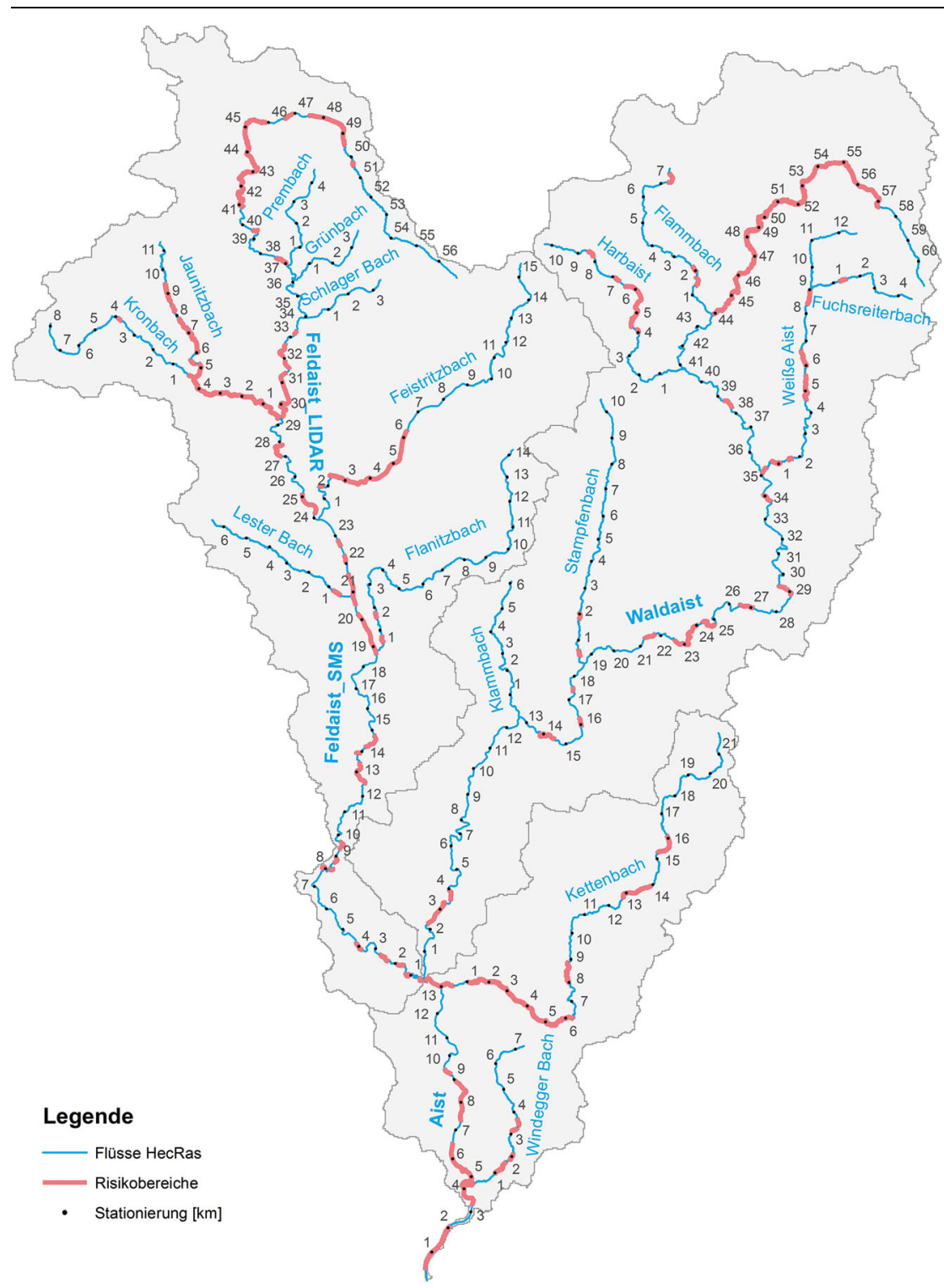

Abb. 8 Darstellung der Risikobereiche für eine potenzielle Versandungsgefahr durch Granitgrus basierend auf den Auswertungen des eindimensionalen Feststofftransportmodells im Einzugsgebiet der Aist

Zustandsklasse nach standardisierter MZB-Methode tritt jedoch erst ab Versandungsklasse 3 ein, wobei in zwei Fällen dieser Versandungsklasse auch noch der gute ökologische Zustand attestiert wurde. Dafür verantwortlich sind strukturelle Feinunterschiede sowie die Vernachlässigung der Parameter Individuendichten bzw. Biomassen innerhalb dieser Bewertungsmethode aufgrund $\mathrm{zu}$ hoher saisonaler Variabilität (Ofenböck et al. 2010). Es zeigte sich, dass die Versandung ein kontinuierlicher Prozess ist, der zu einer schleichenden und stetigen Verschlechterung der ökologischen Gesamtsituation führt noch Einzelfunde dieser Tiere möglich. Auch in der Waldaist waren und sind deutliche Rückgänge zu verzeichnen, in manchen Gewässerabschnitten finden sich aber zumindest noch etwas dichtere Bestände. Diese bestehen jedoch ebenso nur aus Adulttieren, was bedeutet, dass in den letzten Jahrzehnten keine erfolgreiche Reproduktion stattgefunden hat.

Um den Einfluss der Versandung auf den Lebensraum der Flussperlmuschel darzustellen, wurde die Zusammensetzung des Sediments mithilfe der Siebanalysen getrennt nach Ober- und Unterschicht für die unterschiedlichen Flusssysteme analysiert. Starke Feinsedimentbelastungen wurden beispielsweise für Erlau und Kleiner Mühl ausgewiesen. Die Waldaist weist eindeutig gröberes Geschiebe und den geringsten Feinanteil auf. Es kann angenommen werden, dass die etwas bevorzugte Sedimentsituation im Waldaist-System das Überleben der Restbestände der Flussperlmuschel dort begünstigt hat.

Um eine detaillierteres Bild zu erhalten, wurden sowohl der Anteil der feinsten Fraktion $(<0,125 \mathrm{~mm})$ sowie die summierte Sandfraktion bis $2 \mathrm{~mm}$ aus den Deckschicht- bzw. Mischproben nach Flusskilometer dargestellt $(0=$ Mündung). Das Gleiche wurde für die seit dem Jahr 2000 bekannten Muschelfunde bzw. dezidierten NichtNachweise durchgeführt. Damit ergibt sich ein Bild der Änderungen über den Längsverlauf der Untersuchungsgewässer (Abb. 10).

Tendenziell kann aus der Betrachtung der Abschnitte geschlossen werden, dass es einen Zusammenhang zwischen der Ablagerung von Sandund Feinsedimentfraktionen und einem Muschelvorkommen gibt. Dieser ist jedoch nicht zwingend gegeben. Die Gewässersysteme weisen deutliche Unterschiede hinsichtlich der feinsten Fraktion auf und auch lokal ergeben sich hier große Unterschiede (siehe dazu auch Höfler et al. 2016, in diesem Heft). Die noch bekannten Muschelvorkommen sind offenkundig vor allem durch das Fehlen oder einen sehr geringen Anteil an der Fraktion < 0,125 mm determiniert. Mehr als dies durch einen geringen Anteil der Sandfraktion der Fall ist. 


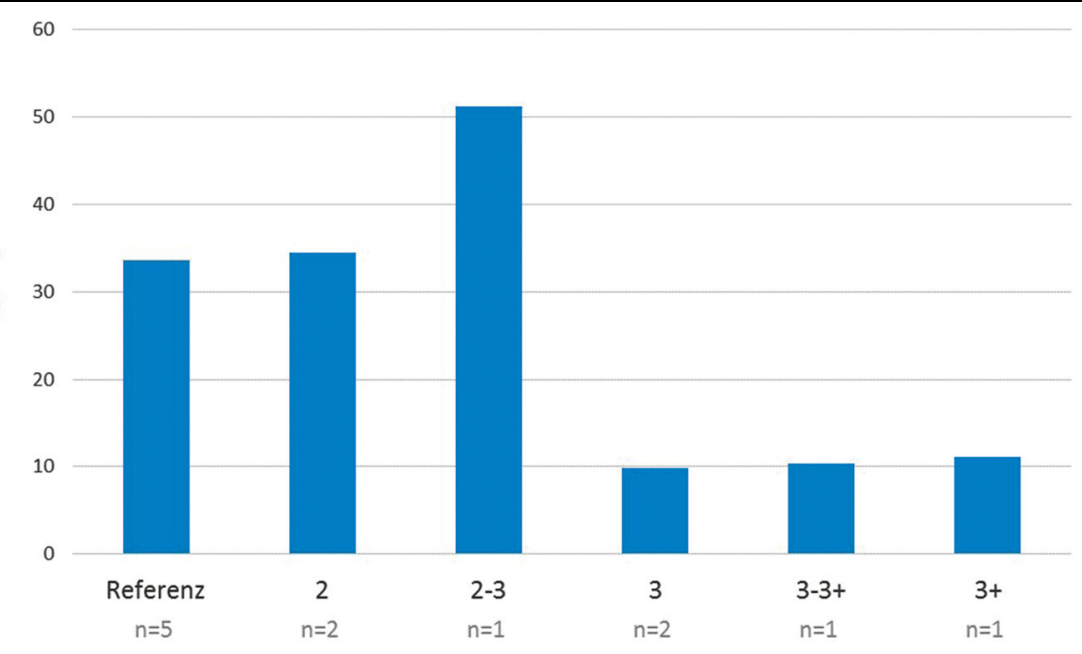

Abb. 9 Mittlere Biomassen [ $\left.\mathrm{g} / \mathrm{m}^{2}\right]$ in Referenz- und versandeten Abschnitten basierend auf einer hochgerechneten geschätzten Substratverteilung der unterschiedlichen Versandungsklassen

\section{Grundsätze für ein zukünftiges Feststoffmanagement im Naturnahen Wasserbau}

Der abschließende Teil des vorliegenden Fachbeitrags fasst alle wesentlichen Ursachen der Versandung der Fließgewässer der Böhmischen Masse zusammen. Ein skalenorientierter Zugang soll die Bedeutung der einzelnen Ursachen im Projektgebiet festlegen. Die Bewertung beinhaltet auch die generelle Aussagekraft, das heißt die Übertragbarkeit zwischen den Flusssystemen, und die Größenordnung in Bezug auf den Anteil an der Versandungsproblematik.

\subsection{Auswirkungen der globalen Erwärmung}

Generell ist festzuhalten, dass klimabedingte Veränderungen der Flussmorphologie sehr stark von den hydrologischen Veränderungen wie beispielsweise der Frequenz der Hochwässer oder Veränderungen des hydrologischen Regimes abhängen. Ashmore und Church (2001) konnten nachweisen, dass Veränderungen in der Flussmorphologie aber auch im Sedimenthaushalt großteils mit Prozessen des „Climate wettings“ in Verbindung stehen und nicht alleine durch „Global Warming“ hervorgerufen werden. Die Auswirkungen der globalen Erwärmung bildeten sich auch im Untersuchungsgebiet durch eine deutliche Zunahme der Starkniederschläge parallel zum Anstieg der Temperatur ab (Hauer et al. 2013). Damit einhergehend zeigen auch die
Fließgewässer im Gebiet eine starke Sensibilität in Hinblick auf die globale Erwärmung. Die Ursachen für die zunehmende Versandung, bedingt durch die globale Erwärmung und das damit in Verbindung stehende Climate Wetting, erfolgt jedoch nicht durch die Zunahme der (Stark-)Niederschläge alleine. Die Ursachen sind auch durch Veränderung der Niederschlags-Abfluss-Verhältnisse begründet (Abb. 11). Lokal auftretende Starkniederschläge verursachen nur eingeschränkt erhöhte Wasserführungen in den Vorflutern. Somit tritt zwar ein erhöhter Feststoffeintrag aus dem betroffenen Detaileinzugsgebiet auf, der Abtransport erfolgt aber nicht daran gekoppelt. Dies auf die transportwirksamen Abflüsse gezeigt werden (Abb. 11). Durch diese Entkoppelung des Eintrags und der Möglichkeiten des Weitertransports ist eine kontinuierliche Akkumulation des Versandungsmaterials im gesamten Einzugsgebiet gegeben.

\subsection{Geologie und Bodenaufbau}

Die geologischen Vorgänge der Verwitterung des Festgesteins sind ein weiterer wichtiger Faktor für das tatsächliche Feststoffaufkommen. Am Institut für Angewandte Geologie (IAG) an der Universität für Bodenkultur Wien wurden die im Untersuchungsgebiet vorkommenden lithologischen Hauptgruppen hinsichtlich ihres Beitrags zum Versandungsrisiko bewertet. Grobkorngneise (z. B. Weinsberger Granit) und Grobkonnte durch Trendanalysen in Bezug korngranite (z. B. grober Perlgneis) wurden als jene geologischen Materialien klassifiziert, welche stark zur Vergrusung neigen und somit einen deutlich höheren Anteil an der Feststoffproduktion beitragen als dies beispielsweise für Feinkorngranite ausgewiesen wurde. Die Verteilung dieser Grobkorngranite und -gneise ist über das gesamte Projektgebiet unterschiedlich. Die Ergebnisse der Kartierung bestätigen dabei den Zusammenhang zwischen geologischem Untergrund und Versandungsneigung der Fließgewässer. Die Einzugsgebiete Kleine Mühl, Kleine Gusen, Teile von Feld- und Waldaist und die Naarn weisen eindeutig einen hohen prozentuellen Anteil an diesen geologischen Elementen auf und sind gleichzeitig von einer starken Versandung betroffen. Große Mühl und Große Rodl, welche über weite Strecken mit Klasse 0 und Klasse 1 bewertet wurden, was einer natürlichen oder naturnahen Sedimentzusammensetzung entspricht, zeigen beispielsweise einen höheren Anteil an Feinkorngraniten und sogenannten Metamorphiten.

Darüber hinaus beeinflusst der geologische Untergrund auch die auftretende Versandungscharakteristik und den Grad der menschlichen Landnutzung. Beispielsweise ist das FeldaistSystem oder auch das System der Großen Gusen, die eigentlich von weniger erosionsanfälligen Feinkorngraniten dominiert sind, von eher sanften Landschaftsformen geprägt, die intensiver vom Menschen für Siedlungszwecke und Landwirtschaft genutzt werden als andere Einzugsgebiete. In diesen Gewässern treten dann häufig neben der Versandung auch vermehrt Feinsedimentprobleme auf. Auch die Charakteristik der Versandung ist eine andere, da der Sand hier sehr fein ist. Es bilden sich oft Dünen aus und die Bedingungen für die aquatische Fauna sind oftmals noch stärker beeinträchtigt als bei grobkörnigeren Verwitterungsprodukten. Ganz deutlich zu beobachten ist diese sehr feine Versandung auch beispielsweise im Kettenbach, einem linksufrigen Zufluss in den Unterlauf der Aist.

Aus der Geologie resultieren auch die bodenkundlichen Bedingungen. Durch das Kristallin bilden sich eher Rohbodentypen mit oftmals sehr dünnen A-Horizonten aus. Werden diese Böden gestört, beispielsweise bei Straßenanschnitten, frischen Böschungen oder bei Waldarbeiten, kommt es oft zu 

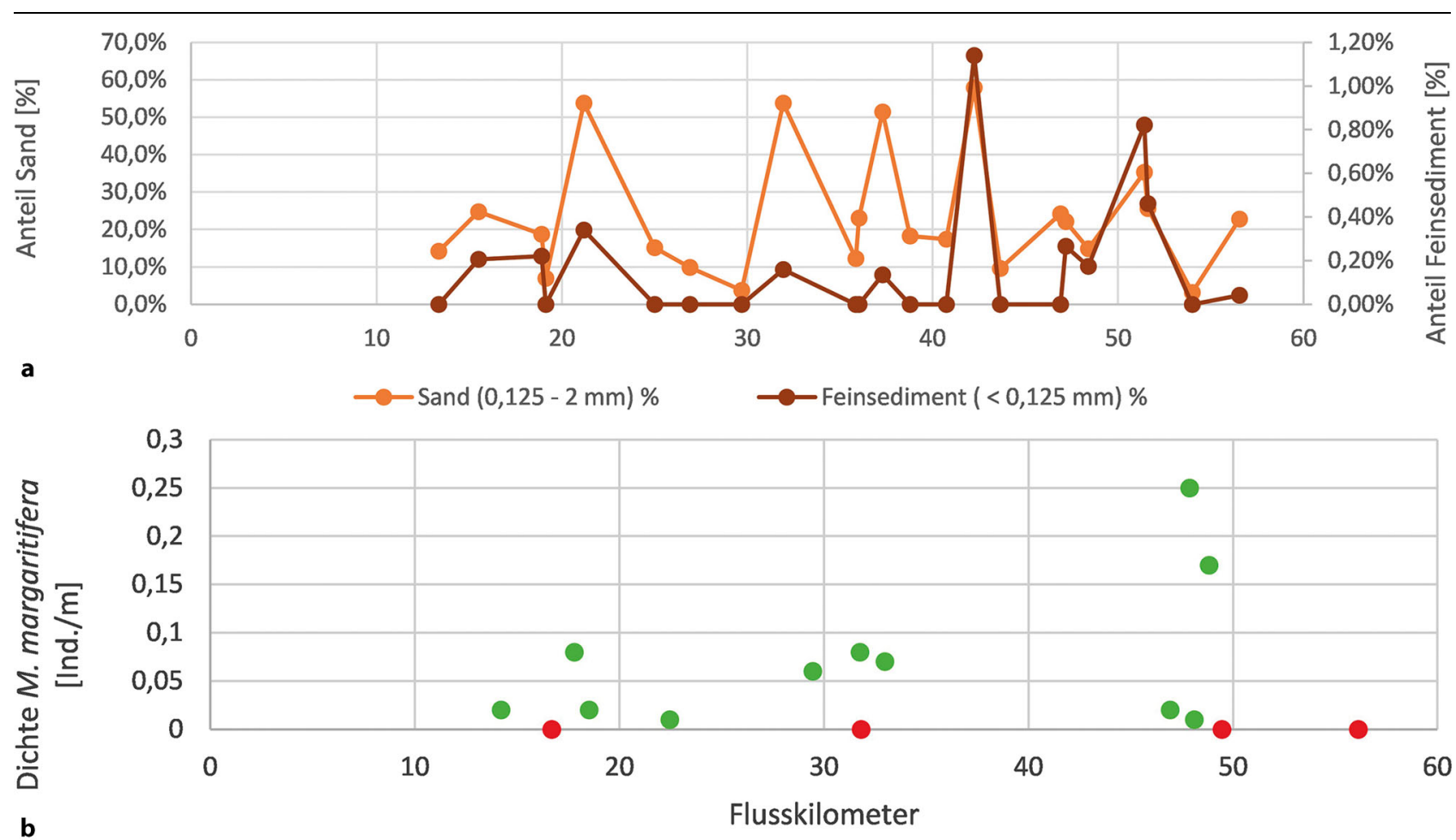

Abb. 10 Situation in der Großen Mühl hinsichtlich feinen Sedimentfraktionen (oben) und der Flussperlmuschelnachweise seit dem Jahr 2000 (unten: rote Punkte: Suche, aber keine Nachweise, grüne Punkte: Nachweise der Flussperlmuschel in Individuen pro Laufmeter Fließgewässer)

langanhaltenden Erosionsprozessen, da eine schützende Vegetationsschicht nur langsam wiederaufkommt.

\subsection{Forstwirtschaftliche Nutzung}

Vor allem die oberen Einzugsgebiete der untersuchten Gewässer werden teilweise sehr intensiv forstwirtschaftlich genutzt (z. B. Naarn, Feldaist, Waldaist). Ein Zusammenhang mit der zunehmenden Versandung muss dabei dringend angenommen werden. Sowohl in den größeren Forstbetrieben wie auch teilweise im eher kleinflächig genutzten bäuerlichen Wald treten aufgrund der Bewirtschaftungsform und der Nutzungsintensität starke Erosionserscheinungen zu Tage. Dies ist auch im bereits erwähnen Bodenaufbau begründet, der einmal angegriffen zu einer langanhaltenden Erosion neigt. Die Auswirkungen ergeben sich aus einer Kombination von nicht standortgerechter Baumartenwahl (hoher Fichtenanteil), negativen Auswirkungen durch die Nutzung als Altersklassenwald mit fehlender Kraut- und Strauchschicht und außerdem relativ großen Kahlschlagflächen, dem zunehmenden Einsatz von schwerem Arbeitsgerät wie Harves- ter und Forwarder und die damit im Zusammenhang stehende deutliche Intensivierung des forstlichen Wegebaus. Die Verbreiterung der Wege sowie die Anlage von Holzlager- und Umkehrplätzen führen zu teilweise großen $\mathrm{Ge}$ ländeanschnitten. Die dazu angelegten Entwässerungssysteme transportieren das erodierte Material direkt in die Gewässer beziehungsweise beschleunigen den Abfluss in die ohnehin erosionsanfälligen Gräben. Darüber hinaus reichen die nicht standortgerechten Forste bis an die Gewässer heran. Die Wurzeln der Fichte bieten aber keine adäquate Boden- und Uferstabilisierung, wodurch teilweise tief ausgerissene Gräben und übermäßige Uferanbrüche als eindeutige Erosionsherde identifiziert werden konnten (Abb. 12).

\subsection{Siedlungsentwicklung und Straßenbau}

In einer weiteren Betrachtung der Umlandnutzung wurden im Einzugsgebiet der Aist in ausgewählten Abschnitten die Entwicklungen der Siedlungsstruktur untersucht. Die flächenhafte Ausweisung der Siedlungsentwicklung im Zeitraum 1950 bis 1990 zeigte die zunehmende Ausdehnung der Wohnund Gewerbeflächen. Durch die Bodenversiegelung und die Fassung bzw. konzentrierte Abfuhr des Niederschlags von diesen Flächen in kleine Zuflüsse der Hauptgewässer kommt es zu einer hydraulischen Überlastung und damit zu einem verstärkten Abtrag von Material in diesen Gräben. Dies tritt vor allem in jenen Bereichen auf, wo die Feld- und Waldaist bereits tief in das Grundgebirge eingeschnitten sind und die Zubringer und Erosionsgräben entsprechende Gradienten aufweisen (> $10 \%$ ). Die Effekte der beschleunigten Abfuhr des Regenwassers werden zusätzlich durch die beschriebenen Auswirkungen der globalen Erwärmung negativ verstärkt. Somit kommt es in der zeitlichen Betrachtung $\mathrm{zu}$ einer Überlagerung zweier sich negativ auf die Versandung auswirkender Prozesse. Mit der Siedlungsentwicklung einhergehend wurde in den letzten Jahrzehnten auch das Straßennetz ausgebaut. Darüber hinaus kam und kommt es durch den vermehrten Einsatz von größeren Maschinen in der Land- und Forstwirtschaft auch im ländlichen Wegenetz zu Ausbauten. Neben den dadurch entstehenden neuen Erosionsherden besteht 


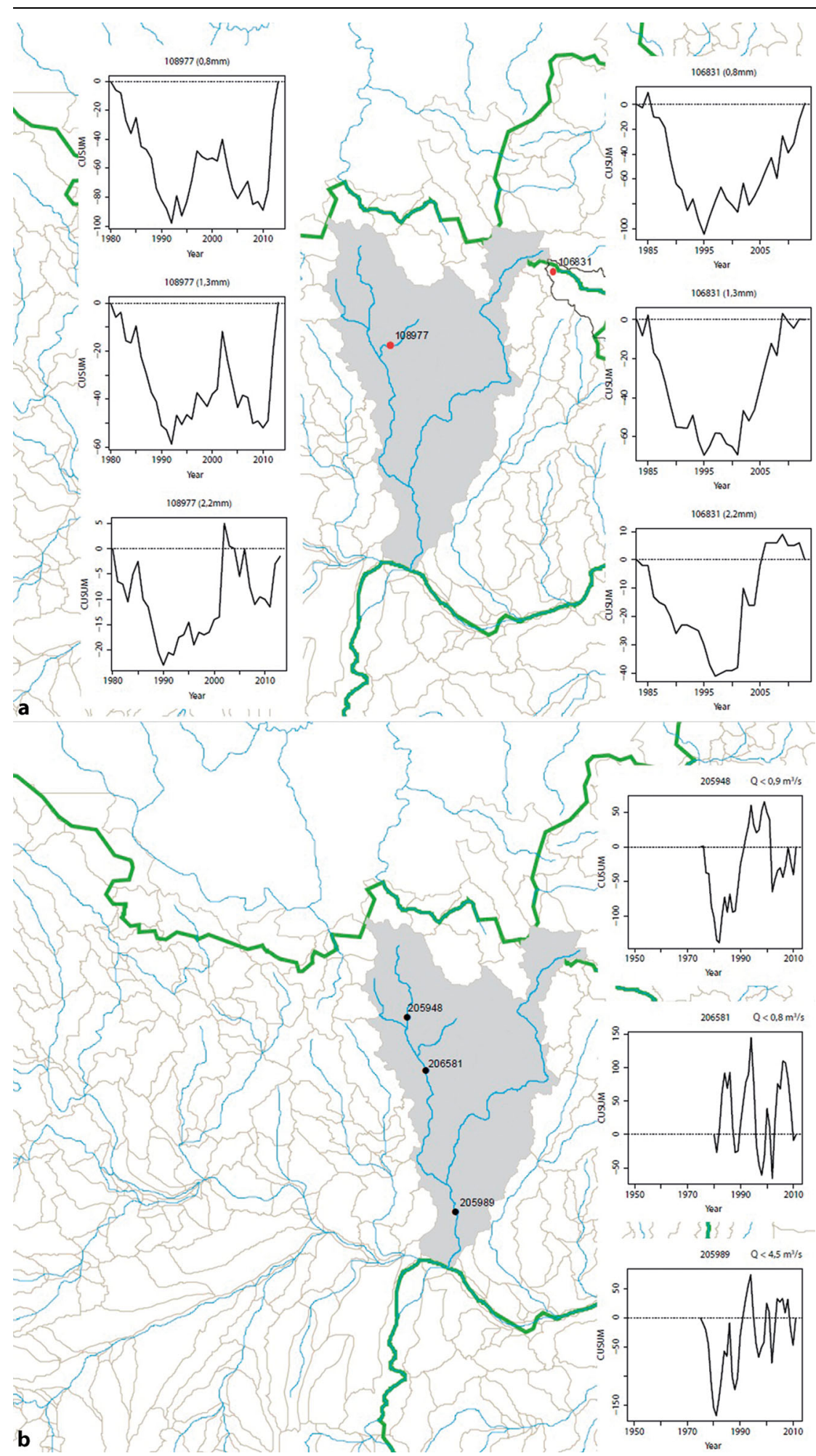

Abb. 11 Kumulative Abweichung vom Mittelwert für Starkniederschläge im Einzugsgebiet der Aist, (b) Kumulative Abweichung vom Mittelwert für die transportwirksamen Abflüsse im Einzugsgebiet der Aist durch die, die Infrastruktur begleitenden, Entwässerungssysteme ein dichtes Netz an Eintragswegen. Damit wird oft auch die Verbindung von weiter weg gelegenen Erosionsquellen zum Gewässer hergestellt.

\subsection{Landwirtschaftliche Nutzung}

Die landwirtschaftliche Nutzung in den Flusseinzugsgebieten der Böhmischen Massen bedarf hinsichtlich ihres Anteils an der Versandungsproblematik einer differenzierten Betrachtung. Ackerflächen, welche intensiv durch teilweise erosionsfördernde Kulturen (z. B. Maisanbau) bewirtschaftet werden, tragen mitunter bei ungünstiger Exposition zu einem deutlichen Feststoffeintrag in die Fließgewässer der Böhmischen Masse bei. Dies betrifft jedoch meist Kornfraktionen deutlich $<1 \mathrm{~mm}$, welche in der Folge als Schwebstoffe im Vorfluter flussab transportiert werden bzw. im Falle von Sedimentation oberflächlich und im Interstitial den Schotterlückenraum verschließen. Die Anteile an den im Projekt zentral untersuchten Grobsand- und Feinkiesfraktionen variieren je Einzugsgebiet, wobei sie generell einen geringen bis mittleren Volumsanteil besitzen (20 bis $40 \%$ ). Ein weiterer Aspekt in der Beurteilung der landwirtschaftlichen Nutzung hinsichtlich ihres Anteils am Feststoffaufkommen ist die Form des landwirtschaftlichen Wasserbaus. Hier konnte in einigen Fallbeispielen über das gesamte Untersuchungsgebiet verteilt, vor allem bei ungünstigem steilen Gelände und einem begradigten Verlauf, offene Entwässerungsgräben als eine lokale Quelle hinsichtlich Feststoffeintrag nachgewiesen werden. Durch die konzentrierte Abflussbildung bei Starkregenereignissen kommt es in diesen Gräben zu erosiven Vorgängen. Mitunter wird die Erosion durch die Tatsache verstärkt, dass diese Rinnen meist nur periodisch Wasser führen und im Winter bei tiefen Temperaturen durch Frostsprengung anstehender Boden bzw. anstehendes Festgestein einer zusätzlichen physikalischen Verwitterung unterliegen.

\subsection{Sedimenttransportverhalten}

Im Projekt wurde des Weiteren das Transportverhalten des Versandungsmaterials im Wasserbaulabor der Universität für Bodenkultur untersucht. Die Versuche, die neben der Bestimmung der Schubgeschwindigkeit, der soge- 

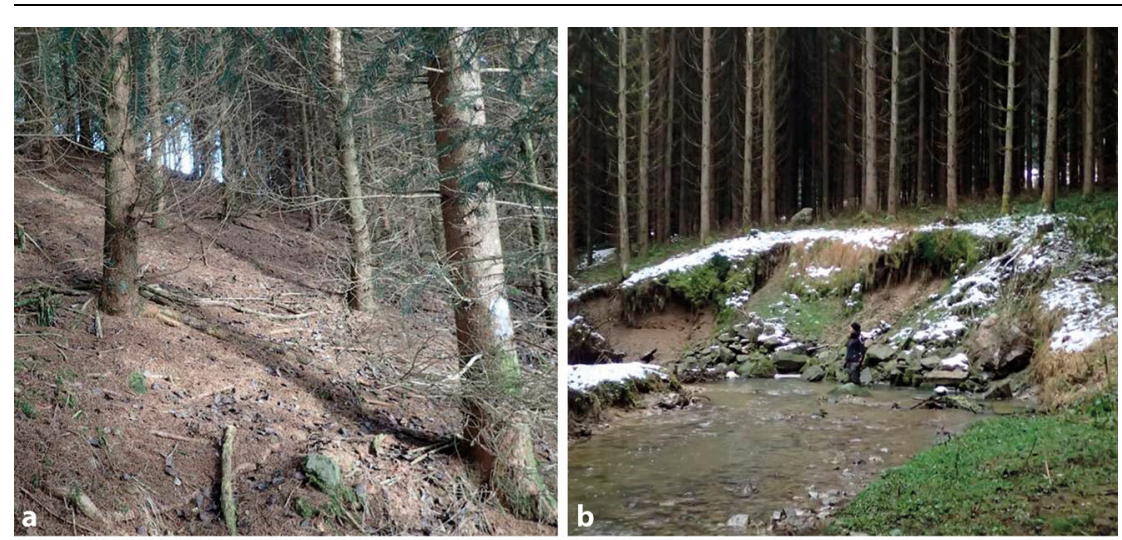

wieder ausgetragen werden und sich im Umland teilweise durch Vegetation unterstützt wieder ablagern. Diese natürlichen Vorgänge wurden durch Regulierungen und Erhaltungsmaßnahmen nach Hochwasserereignissen unterbunden. Die „Selbstreinigungskraft" der Gewässer ging somit oftmals verloren. Deshalb akkumuliert das Material ebenfalls auf unnatürliche Weise im Gewässer.

Weiters wurden durch Regulierungen und Entnahme von großen Blöcken zur Verbesserung der Holztrift, für die Sicherung der Ufer oder aus Hochwasserschutzgründen Strecken mit sehr monotonen Abflussverhältnissen geschaffen. Wie auch im physikalischen Modellversuch gezeigt werden konnte, neigen solche Strecken mit einer geringen Turbulenz zu einer verstärkten Versandung. Der Sand teilt sich hier auch sehr gleichmäßig über das gesamte Profil auf und es bilden sich somit keine unterschiedlichen Choriotope mehr aus. Speziell nach dem Hochwasser 2002 wurden in vielen Bereichen die großen Blöcke aus den Gewässern entfernt, um die Abflussleistung zu erhöhen. Dies geschah zum Teil auch in Bereichen, wo das Umland eher nur extensiv land- und forstwirtschaftlich genutzt ist. Oftmals sind diese Blöcke noch auf gewässernahen Flächen vorhanden und könnten zukünftig für Renaturierungsmaßnahmen genutzt werden.

nannten Versandungsfronten - Sohlformen mit einer steilen Ausprägung im vorderen Bereich - über unterschiedlich rauem Sohlmaterial auch detaillierte Messungen von Fließgeschwindigkeit und Turbulenz beinhalteten, brachten neue Erkenntnisse mit sich, welche für das gesamte Projektgebiet Gültigkeit besitzen. Die Variabilität der kleinräumigen Turbulenz über der Sohle konnte eindeutig mit den unterschiedlichen Geschwindigkeiten der Fortbewegung des Materials in Verbindung gebracht werden (Hauer et al., 2015). Die Rauigkeit und die damit kleinräumig hohe und variable Turbulenz vermindert sich mit zunehmender Überdeckung des gröberen Substrats durch die Sandschichten. Aus diesem physikalisch begründeten Verhalten des Materials im Gewässer ergibt sich folgende zusammenfassende Schlussfolgerung: Je mehr sich von diesen sandigen und feinkiesigen Feststoffen im Gewässer befindet (z. B. vollständige Überdeckung der Sohle), desto schwieriger ist es für den Fluss selbst, unter gleichbleibenden naturräumlichen (hydraulischen) Bedingungen das Material abzutransportieren. Daraus ergibt sich ein weiterer negativer kumulierender Effekt.

\subsection{Defizite im Gewässer-Umland Gefüge und hydromorphologische Überprägung}

Die laterale Konnektivität, also die Verbindung zwischen einem Gewässer und seinem Umland, spielt sowohl für die Strukturvielfalt wie auch für den Sedimenthaushalt eine entscheidende Rolle. Grundsätzlich sind Uferanbrüche in einem gewissen Ausmaß natürlich. Oftmals ist jedoch nur ein sehr dünner oder kein Ufergehölzstreifen vorhanden. Dies ist vor allem in forstlich und landwirtschaftlich intensiv genutzten Bereichen der Fall. Kommt es dort zu Uferanrissen, wird unnatürlich viel Material erodiert. Die Vernetzung zwischen Gewässerumland und Flussschlauch spielt auch deshalb eine große Rolle, weil in unbeeinflussten Fließgewässersystemen Sedimente eigendynamisch

\section{Zusammenfassung und Schlussfolgerungen}

Es zeigt sich, dass die Einflussfaktoren auf den quantitativen Grobsandund Feinkiesanteil in den Gewässern der Böhmischen Masse vielfältig und auf unterschiedlichen flussmorphologischen Skalen zu bewerten sind. Die Ergebnisse der schutzwassertechnischen Untersuchungen zeigten in Abhängigkeit vom Materialeintrag und von den hydraulischen Rahmenbedingungen eine Reduktion der Abflusskapazität, die es im Sinne der Gewährleistung des Hochwasserschutzes zu beobachten gilt. Weiters konnte hinsichtlich der Abiotik festgestellt werden, dass die Ursachen der Versandung eine akkumulierende Wirkung besitzen. Aus Sicht der Gewässerökologie wurde festgehalten, dass (i) ein erhöhter Feinsedimenteintrag nachhaltig negative Effekte auf aquatische Biozönosen (Diversität, Abundanz, Biomasse) zeigt, dass (ii) 
der Feinsedimenteintrag ein kontinuierlicher Prozess ist, der zu einer schleichenden und stetigen Verschlechterung der ökologischen Gesamtsituation führt und mittels Routine-Monitoring erst ab starker Versandung (Versandungsklasse 3) $\mathrm{zu}$ erfassen ist und, dass (iii) kleinräumige Strukturen durch eine weitgehend intakte Ufervegetation und Kleinstrukturen trotz einer generellen Homogenisierung der Substratzusammensetzung in den versandeten $\mathrm{Ab}$ schnitten dazu beitragen können, eine hohe Biodiversität und ökologische Funktionsfähigkeit zu gewährleisten. Hinsichtlich der Ergebnisse der Flussperlmuschel-Untersuchungen kann aus der Betrachtung der Abschnitte tendenziell geschlossen werden, dass es einen Zusammenhang zwischen der Ablagerung von Sand- und Feinsedimentfraktionen und dem Muschelvorkommen gibt. Dieser ist jedoch nicht zwingend gegeben. Die Gewässersysteme weisen deutlich Unterschiede hinsichtlich der feinsten Fraktion auf und auch lokal ergeben sich hier große Unterschiede. Da die Interaktion des Umlands mit den aquatischen Lebensräumen ein ganz wesentlicher Faktor für die ökologische Funktionsfähigkeit ist, stellt sich für zukünftige Managementaufgaben folgende Prioritätensetzung: (i) Vermeidung von weiteren Sandeinträgen aus dem Umland sowie aus der unmittelbaren Uferregion. (ii) Schaffung von kleinräu- migen Habitat-Strukturen im Gewässer, durch natürliche Totholzeinträge aus der Ufervegetation oder anthropogen initiierte Strukturelemente.

Aufbauend auf die Ursachen der Versandung und das Prozessverständnis in den Einzugsgebieten auf unterschiedliche Skalen wurden im Projekt „Feststoffmanagement im Mühlviertel und im Bayerischen Wald“ unterschiedliche Typen von Maßnahmen für die Einzugsgebiete der untersuchten Fließgewässer konzipiert. Details zum Projekt und hinsichtlich der Maßnahmenüberlegungen sind im Endbericht „Feststoffmanagement im Mühlviertel und Bayerischen Wald" nachzulesen (https://www.land-oberoesterreich.gv. at/159006.htm).

Danksagung Die Autoren möchten sich bei den Auftraggebern (Amt der Oö. Landesregierung, Wasserwirtschaftsamt Deggendorf und Bayerisches Landesamt für Umwelt) für die Initiierung und Finanzierung der Studie „Feststoffmanagement im Mühlviertel und Bayerischer Wald" bedanken, ebenso wie für die finanzielle Unterstützung durch das Bundesministerium für Land- und Forstwirtschaft, Umwelt und Wasserwirtschaft (vertreten durch KPC, Dr. Laber) und den Europäischen Fonds für regionale Entwicklung (Interreg-Förderprogramm). Weiters gilt der Dank folgenden Personen aus den unter- schiedlichen Dienststellen, die in dem Projekt mitgewirkt haben: DI Christian Adler, Mag. Josef Aigner, Dr. Peter Anderwald, DI Franz Gillinger, Mag. Stefan Guttmann, Ing. Kurt Hehenwarter, DI Gisela Kangler, Ing. Sabine Kapfer, DI Thomas Kibler, Dr. Gabriele Schwaller und DI Wilhelm Somogyi.

Open access funding provided by University of Natural Resources and Life Sciences, Vienna (BOKU).

\section{Interessenkonflikt}

C. Hauer, S. Höfler, P. Flödl, C. Gumpinger, H. Habersack, J. Holzinger, A. Kloibmüller, P. Leitner, P. Lichtneger, T. Mayer, F. Ottner, D. Riechl, B. Wagner, T. Walter, F. Weingraber und W. Graf geben an, dass kein Interessenkonflikt besteht.

Open Access Dieser Artikel wird unter der Creative Commons Namensnennung 4.0 International Lizenz (http:// creativecommons.org/licenses/by/4. 0/deed.de) veröffentlicht, welche die Nutzung, Vervielfältigung, Bearbeitung, Verbreitung und Wiedergabe in jeglichem Medium und Format erlaubt, sofern Sie den/die ursprünglichen $\mathrm{Au}$ tor(en) und die Quelle ordnungsgemäß nennen, einen Link zur Creative Commons Lizenz beifügen und angeben, ob Änderungen vorgenommen wurden. .
Ashmore, P., Church, M. (2001): The impact of climate change on rivers and river processes in Canada, Geological Survey Of Canada 555. Ottawa. $58 \mathrm{~S}$

BMLFUW (Hrsg.) (2010): Leitfaden zur hydromorphologischen Zustandserhebung von Fließgewässern, Wien, $71 \mathrm{~S}$.

Cline, L. D., Short, R. A., Ward, J. V. (1982): The influence of highway construction on the macroinvertebrates and epilithic algae of a high mountain stream. Hydrobiologia, 96: 149-159.

Culp, J. M., Davies, R. W. (1985): Responses of benthic macroinvertebrate species to manipulation of interstitial detritus in Carnation Creek British Columbia. Canadian Journal of Fisheries and Aquatic Sciences, 42: 139-146.

Dudgeon, D., Arthington, A. H., Gessner, M. O. et. al. (2006): Freshwater biodiversity: importance, threats, status and conservation challenges. Biological Reviews, 81: 163-182.

Eriksen, C. H. (1966): Ecological significance of respiration and substrate for burrowing Ephemeroptera. Canadian Journal of Zoology, 46 93-103.

Erman, D. C., Ligon, F. K. (1988): Effects of discharge fluctuation and the addition of fine sediment on stream fish and macroinvertebrates below a water-filtration facility. Environmental Management, 12: 85-97.
Europäische Union (EU) (Hrsg.) (2000): Richtlinie 2000/60/EG des Europäischen Parlaments und des Rates vom 23. Oktober 2000 zur Schaffung eines Ordnungsrahmens für Maßnahmen der Gemeinschaft im Bereich der Wasserpolitik. Flödl, P. (2015): Möglichkeiten des naturnahen Wasserbaus in Bezug auf die Versandungsproblematik von Fließgewässern der Böhmischen Masse. Masterarbeit - Institut für Wasserwirtschaft, Hydrologie und konstruktiver Wasserbau (IWHW), BOKU-Universität für Bodenkultur

Hauer, C. (2015): Review of hydro-morphological management criteria on a river basin scale for preservation and restoration of freshwater pearl mussel habitats. Limnol. - Ecol. Manag. Inland Waters, The current status and future challenges for the preservation and conservation of freshwater pearl mussel habitats, 50: 40-53. doi:10.1016/j.limno.2014.11.002

Hauer, C., Unfer, G., Tritthart, M., Habersack,

H. (2011): Effects of stream channel morphology, transport processes and effective discharge on salmonid spawning habitats. Earth Surface Processes and Landforms, 36(5): 672-685.

Hauer, C., Unfer, G., Holzmann, H., Schmutz, S.

Habersack, H. (2013): The impact of discharge change on physical instream habitats and its response to river morphology. Climate Change, 116(3-4): 827-850.
Hauer, C., Höfler, S., Dossi, F., Flödl, P., Graf, G., Graf, W., Gstöttenmayr, D., Gumpinger, C., Holzinger, J., Huber, T., Janecek, B., Kloibmüller, A., Leitner, P., Lichtneger, P., Mayer, T., Ottner, F., Riechl, D., Sporka, F., Wagner, B., Habersack, H. (2015): Feststoffmanagement im Mühlviertel und im Bayerischen Wald. Endbericht. Studie im Auftrag des Amtes der Oberösterreichischen Landesregierung, gefördert durch das BMLFUW und das Interreg Programm Bayern - Österreich 2007 - 2013. Wien, $391 \mathrm{~S}$

Hjulström, F (1935): Studies of the morphological activity of rivers as illustrated by the River Fyris: Inaugural Dissertation. Almqvist \& Wiksells. Höfler S., Gumpinger C. (2014): Erhebung der Feinsedimentbelastung in oberösterreichischen Alpenvorland-Gewässern - Inklusive Literaturstudie zum Thema Feinsediment in Gewässerökosystemen. - Im Auftrag des Amtes der Oö. Landesregierung Direktion Umwelt und Wasserwirtschaft, Abteilung Oberflächengewässerwirtschaft, Wels, $106 \mathrm{~S}$.

Höfler, S., Gumpinger, C., Hauer, C. (2016): Ökologische Maßnahmen an kleinen und mittelgroßen Fließgewässern Auswirkungen auf die Qualitätselemente der Europäischen Wasserrahmenrichtlinie und Grenzen der Wirksamkeit unter besonderer Berücksichtigung der Feinsedimentproblematik. Österreichische Wasserund Abfallwirtschaft. 


\section{Originalarbeit}

Kellerhals, R. (1967): Stable Channels with Gravel-Paved Beds. J. Waterw. Harb. Div. 93: 63-84.

LAWA (2000): Arbeitskreis GewässerbewertungFließgewässer: Gewässerstrukturgütekartierung in der Bundesrepublik Deutschland: Verfahren für kleine und mittelgroße Fließgewässer. Kulturbuch-Verlag, Schwerin.

Leitner, P., Hauer, C., Ofenböck, T., Pletterbauer, F., Schmidt-Kloiber, A., Graf, W. (2015) Fine sediment deposition affects biodiversity and density of benthic macroinvertebrates: A case study in the freshwater pearl mussel river Waldaist (Upper Austria). Limnol. - Ecol. Manag. Inland Waters, The current status and future challenges for the preservation and conservation of freshwater pearl mussel habitats 50: 54-57. doi:10.1016/j.limno.2014.12.003.

Leopold, L., Maddock, T. (1953): The Hydraulic Geometry of Stream Channels and Some Physiographic Implications. Y Rep.
Leopold, L. B, Wolman, M. G, Miller, J. P (1964): Fluvial Processes in Geomorphology.

Montgomery, D. R., Buffington, J. M. (1997): Channel-reach morphology in mountain drainage basins. Geological Society American Bulletin, 109: 596-611.

Neuhold, C., Stanzel, P., Nachtnebel, H. P. (2009): Incorporating river morphological changes to flood risk assessment: uncertainties, methodology and application. Nat. Hazards Earth Syst. Sci. 9: 789-799.

Nixon, M., LACEY, INGLIS (1959): A Study of the bank-full discharges of rivers in England and Wales. ICE Proc. 12, 157-174. doi:10.1680/ iicep.1959.12123

Ofenböck, T., Moog, O., Hartmann, A., Stubauer, I. (2010): Leitfaden zur Erhebung der Biologischen Qualitätselemente, Teil A2 - Makrozoobenthos. Bundesministerium für Land- und Forstwirtschaft, Umwelt und Wasserwirtschaft. $211 \mathrm{~S}$.

Ofenböck, T., Graf, W., Leitner, P. (2008): Eurolimpacs - Deliverable No. 224. Report - Effect of siltation on mountain rivers (Task 2.2).

Rosenberg, D. M., Wiens, A. P. (1978): Effects of sediment addition on macrobenthic invertebrates in a northern Canadian stream. Water Research 12: 753-763.

Ward, J. V., Stanford, J. A. (1995): Ecological connectivity in alluvial river ecosystems and its disruption by flow regulation. Regulated Rivers, Research and Management, 11: 105-119.

Wood, P. J., Armitage, P. D. (1997): Biological effects of fine sediment in the lotic environment. Environ. Management 21: 203-217. 